Article

\title{
Osa-miR820 regulatory node primes rice plants to tolerate salt stress in an agronomically advantageous manner
}

\author{
Neha Sharma, Sudhir Kumar and Neeti Sanan-Mishra* \\ 1 Plant RNAi Biology Group, International Centre for Genetic Engineering and Biotechnology, Aruna Asaf Ali Marg, New \\ Delhi-110067; royaldna@gmail.com; sudheerbgs20@gmail.com: neeti@icgeb.res.in \\ * Correspondence: neeti@icgeb.res.in; Tel. (O): +91-11-2674 1358/61; Fax (O): +91-11-2674 2316
}

\begin{abstract}
Plant microRNAs (miRs) play an important role in regulating gene expression under normal and stressful environments. Here we report the functional implications on the role of Osa-miR820, which can be grouped as a young, rice-specific miR. It is a member of the class II transposon-derived small RNA family and is processed as 21-nt and 24-nt length variants, respectively. Size of the miR820 family varies from 1 to 16 across the Oryza AA genomes. The 21-nt Osa-miR820 negatively regulates a de novo methylase, OsDRM2 (domains rearranged methyl transferase) that prevents methylation of the CACTA transposon loci in the rice genome. In an earlier report we have detailed the expression profiles of Osa-miR820 and its target in abiotic stress responses using rice varieties exhibiting varying response to salt stress. In this study, artificial miR based approach was employed to specifically overexpress 21-nt Osa-miR820 in rice plants (OX-820). These plants exhibited enhanced vigour, $\sim 25-30 \%$ increase in the number of spikelets per panicle and increased grain filling, under normal and salt stress conditions. The OX-820 lines showed a better water use efficiency and higher proline accumulation under salt stress. These plants can serve as a useful source for dissecting the molecular machinery governed by Osa-miR820:DRM2 node to prime tolerance to salt stress in an agronomically advantageous manner.
\end{abstract}

Keywords: rice; salt stress; artificial microRNA; over-expression; panicles

\section{Introduction}

The sessile nature of plants warrants a continued interaction with their external environment for optimal growth and development. This is a multifarious activity that necessitates intricate coordination of gene expression at the transcriptional and post-transcriptional levels. The microRNAs (miRs) are endogenously transcribed regulatory small RNAs, which act in an exceedingly precise manner to rapidly adjust changes in gene expression. They regulate almost every aspect of plant biology such as root and shoot initiation, leaf development, organogenesis, phase transition, flower morphogenesis, phytohormone response and so on [1-6]. The role of miRs in synchronizing plant responses to biotic and abiotic stresses is well recognized [7-9] and has been extensively studied in model plants, Arabidopsis [5, 7,9] and rice [8, 10-13]. New insights into the expression profiles and regulation of miRs have resulted from the application of deep-sequencing technologies and largescale small RNA expression profiling [14-18]. Using the approach of comparative miR profiling followed by experimental validation, our group profiled rice miRs that show tissue-preferential expression patterns [2] and mapped the changes in their profiles in response to salt stress using different rice genotypes $[13,19]$.

Studies involving miR over-expression also highlighted their crucial roles in regulating gene expression for optimal development and response to stress. It was shown that the expression of miR395 was induced under conditions of sulphate limitation to regulate a high-affinity sulphate transporter and three ATP sulfurylases (ATPS) so that plants could survive in low sulphur containing 
soils [20]. Overexpression of Osa-miR395h in tobacco impaired sulphate homeostasis and distribution in the transgenic plants [21]. It was also reported that osa-miR156 could negatively control the number of panicle branches and grain yield through its target, OsSPL14 [22]. In addition, pollenspecific miRs along with specific siRNAs were proposed to be key regulators in the phase transition from bicellular pollen to tricellular pollen stage [23]. These observations suggest a role for miRs in controlling plant architecture and yield.

Over-expression of stress regulated miRs revealed their additional functions by generating visible plant phenotypes. The drought responsive Osa-miR393 regulates two auxin receptor genes Osa-TIR1 and Osa-AFB2 [24]. Its overexpression resulted in plants that were susceptible to drought and salt stresses [25]. Interestingly these plants showed increased tillering and early flowering [26]. Another example is provided by Osa-miR396d which targets the growth regulatory factor (grf) gene to regulate floral organ identity and husk opening [27]. The over expression of miR396 in tobacco confirmed its important roles in drought tolerance and leaf development [28]. Likewise it was seen that overexpression of high-temperature-responsive Osa-miR397 modulates the expression of Lascorbate oxidase $[29,30]$, resulting in increased grain size, more rice panicle branching and higher grain productivity [31].

The present study is focused on Osa-miR820 to understand its physiological and functional relevance in rice. It is a young, rice specific miR, that was first reported in undifferentiated embryogenic rice callus tissues as miR583 [32]. Later it was proposed to be a member of the class II transposon derived small RNA family, with its expression being controlled epigenetically at its own locus [33, 34]. Osa-miR820 was shown to be down-regulated under drought [35], salt [흐] and arsenic [37] stress. Size of the miR820 family varies from 1 to 16 across the Oryza AA genomes [38] and there are three members in the indica genome [36]. Moreover, 21-nt and 24-nt length variants have also been proposed for Osa-miR820 family in rice, which are processed by the action of the DCL-1 and DCL-3, respectively [39], suggesting its dual mode of action. The 21-nt Osa-miR820 negatively regulates a de novo methylase, OsDRM2 (domains rearranged methyl transferase) in 'trans' to prevent methylation of the CACTA transposon loci in the rice genome [39]. The 24-nt variant act to establish a regulatory loop by controlling epigenetic modifications of OsDRM2 as well as its own locus [34]. These findings indicate a complex regulatory phenomenon governed by the miR family.

Our group has previously described the detailed expression profiles of Osa-miR820 and its target in different tissues of salt-tolerant and salt-susceptible varieties of rice under normal and abiotic stress (salt, high temperature and drought) conditions [36]. The regulatory role of this miR on DRM2 transcripts was evident and the narrow windows of transcript regulation were captured. For gaining further insights into the biological significance of 21-nt species of Osa-miR820 in rice, artificial miR based over-expressing transgenic lines, OX-820 were generated [40]. In the present work, we report the phenotype and physiology of OX-820 under normal and saline conditions.

\section{Results}

\subsection{Osa-miR820 mapped to QTLs associated with agronomic parameters}

To gain insight into the putative role of Osa-miR820, its association with rice QTLs was searched in silico. QTL is a large genomic region controlling a specific trait in plants. Earlier studies have correlated the expression of QTL associated miRs with traits related to agronomic performance [41]. Thus, all QTLs overlapping with the three pri-miR820 loci (coding for miR820a, b and c) on rice chromosomes 1, 7 and 10 were identified and mapped (Supplementary Fig S1).

This analysis resulted in the identification of 55, 28 and 68 QTLs mapping to Osa-miR820a, OsamiR820b and Osa-miR820c loci, respectively. The trait association of QTLs with Osa-miR820 loci is depicted in an interaction map shown in Figure 1A and the list of Osa-miR820 associated QTLs is provided in Table 1. Highest trait density correlated with Osa-miR820b on chromosome 7, followed by Osa-miR820a on chromosome 1 and then with Osa-miR820c on chromosome 10. All three loci of Osa-miR820 showed association with 2 QTLs linked to the trait names 'Days to heading' and 'Plant 
height', respectively. Among the identified QTLs, 11 showed association with more than one trait. Category wise distribution of traits showed that the category 'Yield' was maximally represented followed by 'Vigor'. This indicates that Osa-miR820 family could be functionally associated with the two growth parameters.

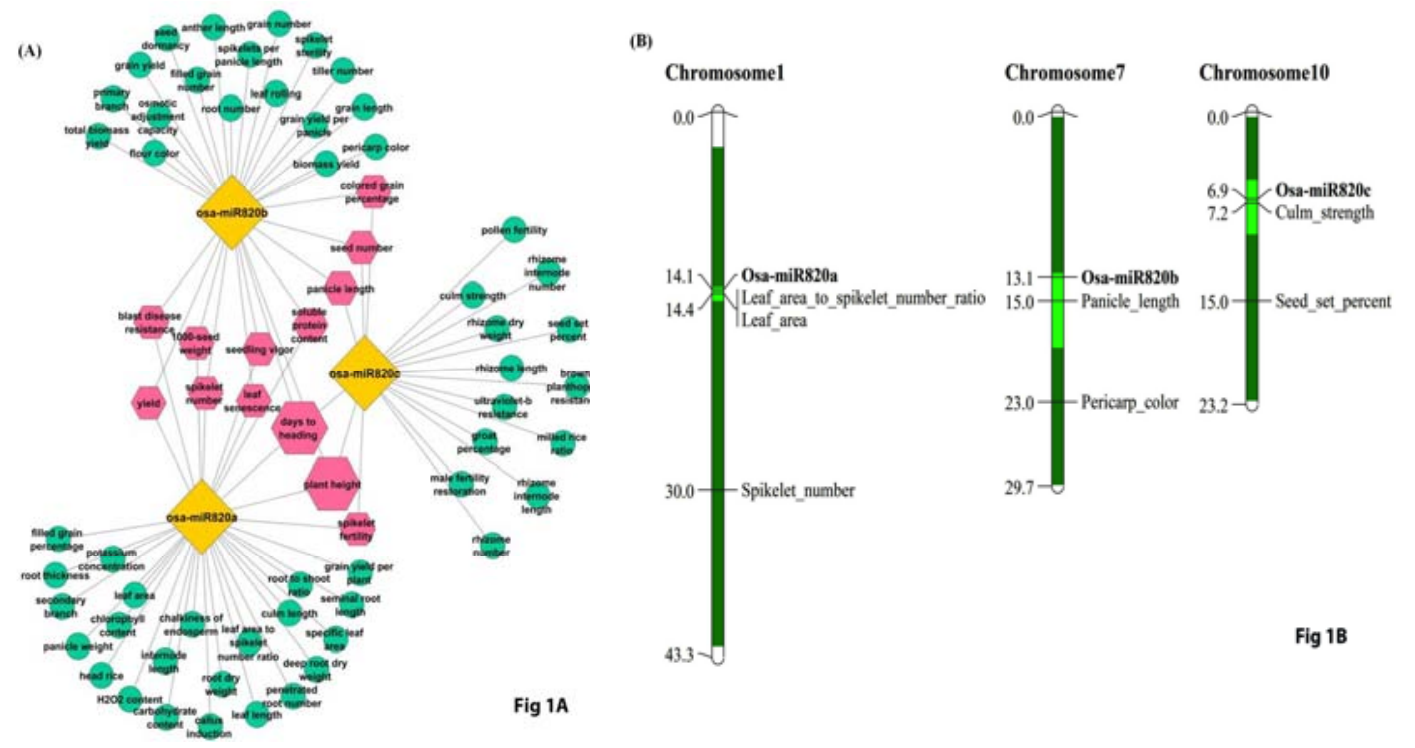

Figure 1. Mapping of QTL associated traits to miR820 locus A) Cytoscape based interaction map. Yellow boxes denote the miR family member; green boxes denote the trait for the associated QTL; Pink hexagons denote QTLs associated with more than one miR820 family member. B) Karyoview of largest and smallest QTLs identified for Osa-miR820a, b and c.

Table 1. Trait wise listing of number of QTLs associated with Osa-miR820 locus

\begin{tabular}{|c|c|c|c|}
\hline Trait category & $\begin{array}{c}\text { Chromosome 1 } \\
\text { (Osa-miR820a) }\end{array}$ & $\begin{array}{c}\text { Chromosome 7 } \\
\text { (Osa-miR820b) }\end{array}$ & $\begin{array}{c}\text { Chromosome 10 } \\
\text { (Osa-miR820c) }\end{array}$ \\
\hline Yield & 19 & 18 & 5 \\
\hline Vigor & 8 & 18 & 2 \\
\hline Development & 6 & 13 & 2 \\
\hline Anatomy & 8 & 6 & 4 \\
\hline Quality & 2 & 8 & 0 \\
\hline Biochemical & 3 & 1 & 2 \\
\hline Abiotic stress & 1 & 2 & 4 \\
\hline Sterility or fertility & 1 & 1 & 3 \\
\hline Biotic stress & Ratio of leaf area to & Panicle length & Culm length \\
\hline Trait for smallest QTL & spikelet number & & \\
\hline
\end{tabular}

To be more specific in predicting the traits associated with Osa-miR820 function, the smallest QTL on each locus was identified (Fig. 1B). This analysis identified "ratio of leaf area to spikelet number", "panicle length" and "culm length" as the associated traits (Table 1). These traits define agronomic parameters that can directly or indirectly affect the rice crop yield. Thus, it was speculated that Osa-miR820 might have a role in regulating plant biomass and/or productivity. 


\subsection{OX-820 rice plants exhibit increased tillering, panicle vigour and enhanced grain yield.}

The presence of two length variants of Osa-miR820 of 21-nt and 24-nt, respectively having common targets presented an interesting challenge in understanding the functional significance of this $\mathrm{miR}$ in the biology of the rice plant. Therefore, artificial miR based strategy was adopted to specifically over-express the 21 -nt length variants. The Osa-miR820 overexpressing transgenic lines (OX-820) were generated and confirmed, as described earlier [40].
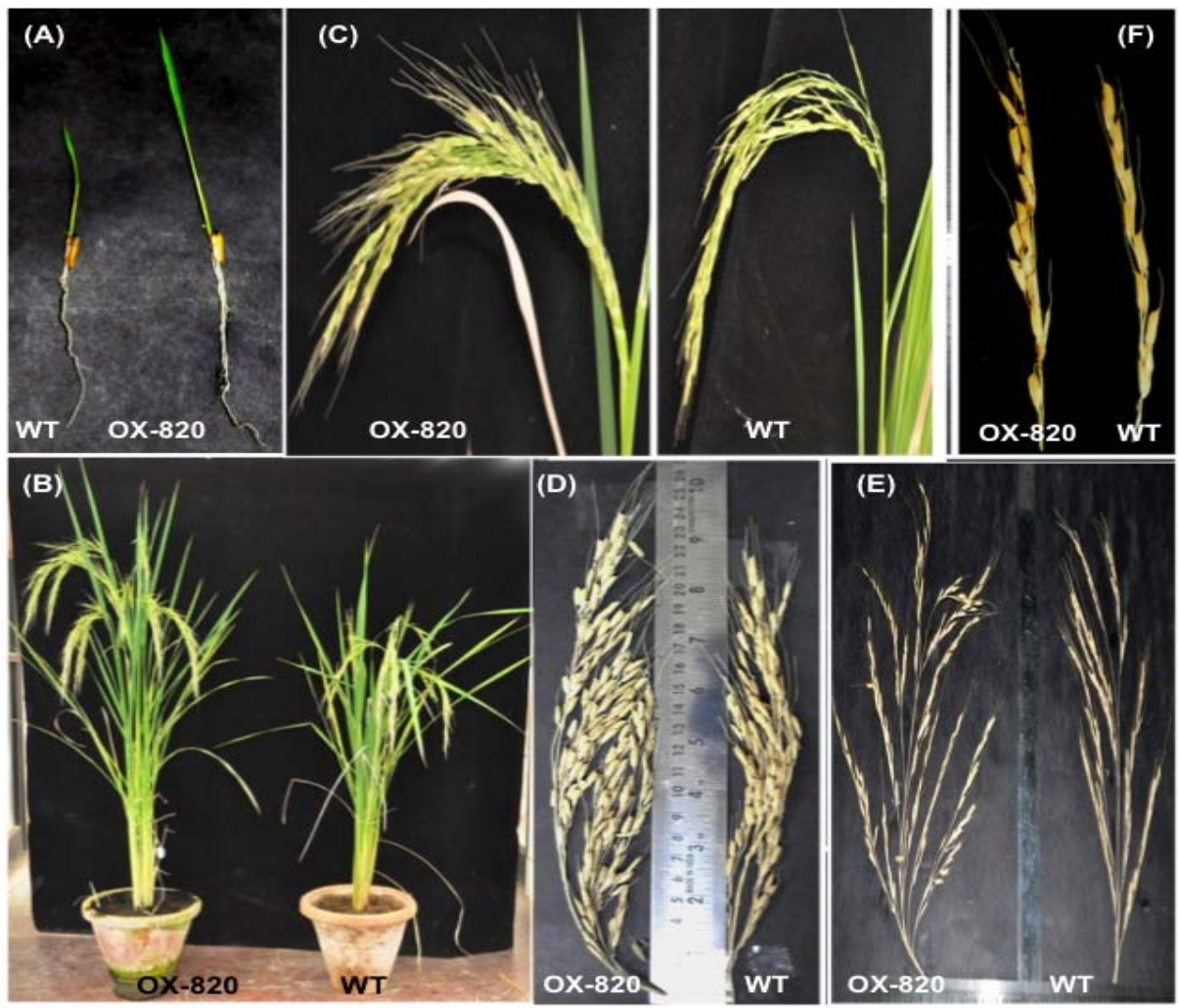

Figure 2. Phenotype of OX-820 rice plants as compared with wild type (WT) (A) 10-day old rice seedlings (B) Plants at the maturity stage (C) Panicles at early grain filling stage (D) Panicle length (E) Panicle branching and (F) Grain filling in an individual spikelet.

For phenotypic comparisons, healthy mature seeds of OX-820 and wild type (WT) were grown on germinating sheets in greenhouse conditions. It was observed that OX-820 seedlings were relatively longer and healthier as compared to WT (Fig. 2A). The increase in length was also evident on comparing the roots (Fig. 2A). The increase in OX-820 plant height was observed in the mature plants as well (Fig. 2B), but the plants exhibited a decrease in total biomass dry weight (Table 2). On comparing the leaf characteristics, it emerged that the leaf length was more in OX-820 plants as compared to the WT but there was no change in the leaf width (Table 2). The OX-820 rice plants showed stouter roots with overall decrease in root length but slightly increased root dry weight (Table 2).

At maturity the OX-820 rice plants had more number of primary tillers (Fig. 2B) and robust panicles (Fig. 2C), when assessed with respect to the WT. The increase in number of tillers was in direct correlation with the number of panicles (Table 3). Analysis of individual panicles revealed that in OX-820 panicles were longer (Fig. 2D), profusely branched on their primary and secondary axis (Fig. 2E) and exhibited denser grain filling (Fig. 2F). This resulted in increased drooping of panicles at the post-anthesis stage in OX-820 plants (Fig. 2C). Thus, it was evident that constitutive over- 
expression of the 21-nt sequence of Osa-miR820 generated a distinct phenotype in rice indicating its role in modulating plant architecture.

Table 2. Comparison of vegetative growth related traits in OX-820 transgenic and WT plants

\begin{tabular}{|l|c|c|c|c|c|c|}
\hline \multicolumn{1}{|c|}{ Line } & $\begin{array}{c}\text { Primary tillers } \\
\text { (number) }\end{array}$ & $\begin{array}{c}\text { Leaf } \\
\text { length } \\
(\mathrm{cm})\end{array}$ & $\begin{array}{c}\text { Mature } \\
\text { plant height } \\
(\mathrm{cm})^{\# \#}\end{array}$ & $\begin{array}{c}\text { Plant biomass } \\
\text { dry weight } \\
(\mathrm{g})\end{array}$ & $\begin{array}{c}\text { Mature } \\
\text { root length } \\
(\mathrm{cm})\end{array}$ & $\begin{array}{c}\text { Mature root } \\
\text { dry weight } \\
(\mathrm{g})^{\sharp \#}\end{array}$ \\
\hline WT-1 & 6 & $45.0 \pm 1.3$ & $34.5 \pm 1.4$ & $26.0 \pm 0.5$ & $25.5 \pm 1.3$ & $4.38 \pm 0.1$ \\
\hline WT-2 & 5 & $42.8 \pm 1.2$ & $33.7 \pm 1.3$ & $25.4 \pm 0.5$ & $23.6 \pm 1.2$ & $4.0 \pm 0.1$ \\
\hline WT-3 & 5 & $44.2 \pm 1.3$ & $31.9 \pm 1.3$ & $25.8 \pm 0.5$ & $24.7 \pm 1.2$ & $4.25 \pm 0.1$ \\
\hline OX-820-L1 & 8 & $59.5 \pm 1.8$ & $37.4 \pm 1.5$ & $21.5 \pm 0.4$ & $22.2 \pm 1.1$ & $4.7 \pm 0.1$ \\
\hline OX-820-L2 & 7 & $56.7 \pm 1.7$ & $39.6 \pm 1.6$ & $18.0 \pm 0.6$ & $25.4 \pm 0.9$ & $5.02 \pm 0.1$ \\
\hline OX-820-L3 & 8 & $56.0 \pm 1.7$ & $38.8 \pm 1.5$ & $23.5 \pm 0.5$ & $21.0 \pm 1.3$ & $4.8 \pm 0.1$ \\
\hline OX-820-L4 & 8 & $54.0 \pm 1.6$ & $38.3 \pm 1.5$ & $22.2 \pm 0.4$ & $19.5 \pm 1.0$ & $4.28 \pm 0.1$ \\
\hline
\end{tabular}

\# Average values per plant; \#\# Values rounded off to one decimal place

High tillering capacity is an important agronomic trait in cereals and it has a positive correlation with grain yield in rice. However higher number of tillers tends to create an imbalance of photosynthetic assimilates among vegetative and reproductive parts, which may compromise productivity $[42,43]$. The OX-820 transgenic plants were compared for agronomic parameters to evaluate their grain productivity (Table 3). It was observed that each primary tiller contained a dense panicle with higher number of spikelets as compared to WT. There was $25-30 \%$ increase in the number of spikelets per panicle in OX-820 plants. This increase was largely due to enhancement in the length and branching of panicles. The spikelets showed increased grain filling resulting in $50-60 \%$ increase in the number of grains (Table 3). The individual grain width remained unaffected and there was a marginal increase in grain length. Thus, constitutive over-expression of the 21-nt sequence of Osa-miR820 resulted in increase in number of spikelets and improvement in grain filling per panicle leading to enhanced grain yield.

Table 3. Comparison of agronomic traits in OX-820 transgenic and WT plants

\begin{tabular}{|c|c|c|c|c|c|c|c|c|}
\hline $\begin{array}{c}\text { Line } \\
\text { number }\end{array}$ & $\begin{array}{c}\text { No. of } \\
\text { panicles }^{\#}\end{array}$ & $\begin{array}{c}1^{\circ} \text { branches } \\
\text { per } \\
\text { panicle }\end{array}$ & $\begin{array}{l}\text { Panicle } \\
\text { length } \\
(\mathrm{cm})^{\# \$}\end{array}$ & $\begin{array}{c}\text { No. of } \\
\text { spikelets per } \\
\text { panicle }^{\sharp \$}\end{array}$ & $\begin{array}{l}\text { Total no. } \\
\text { of grains }\end{array}$ & $\begin{array}{c}\text { 1000-seed } \\
\text { weight } \\
(\mathrm{g})^{\# \$}\end{array}$ & $\begin{array}{l}\text { Grain } \\
\text { length } \\
(\mathrm{cm})^{\# \#}\end{array}$ & $\begin{array}{l}\text { Grain } \\
\text { width } \\
(\mathrm{cm})^{\# \#}\end{array}$ \\
\hline WT-1 & 6 & 6 & $24.1 \pm 0.5$ & $136 \pm 6.8$ & $780 \pm 31$ & $20.9 \pm 0.8$ & 10.0 & 2.1 \\
\hline WT-2 & 5 & 5 & $24.9 \pm 0.5$ & $131 \pm 6.6$ & $647 \pm 26$ & $19.8 \pm 0.8$ & 10.1 & 2.2 \\
\hline WT-3 & 6 & 6 & $23.7 \pm 0.5$ & $128 \pm 7.2$ & $804 \pm 32$ & $20.2 \pm 0.8$ & 10.9 & 2.2 \\
\hline OX-820-L1 & 9 & 5 & $28.2+0.5$ & $159 \pm 8.0$ & $1104 \pm 44$ & $23.4 \pm 0.9$ & 11.0 & 2.1 \\
\hline OX-820-L2 & 8 & 8 & $29.5 \pm 0.6$ & $168 \pm 8.4$ & $1206 \pm 48$ & $23.2 \pm 0.9$ & 11.65 & 2.4 \\
\hline OX-820-L3 & 9 & 7 & $26.4 \pm 0.5$ & $171 \pm 8.5$ & $1373 \pm 55$ & $22.6 \pm 0.9$ & 10.8 & 2.3 \\
\hline
\end{tabular}

\# Average values per plant; \#\# Average values of 10 seeds per plant; \$ Values rounded off to one decimal place

\subsection{Overexpression of Osa-miR820 does not influence response to salt stress}

Earlier studies have shown that Osa-miR820 levels were down regulated under salt stress [흐], so it was apparent to evaluate the effect of salt stress on OX-820 plant phenotype and physiology. To understand the response of OX-820 plants to salt stress the chlorophyll retention assay [44], was performed. Discs cut from healthy leaves of both OX-820 and WT plants were kept in different concentrations of $\mathrm{NaCl}$ and monitored at regular time intervals. Discs kept in deionized water served 
as control. After $72 \mathrm{~h}$, the leaf discs were collected (Fig. 3A) and the chlorophyll content was quantitated and plotted (Fig. 3B). Significant difference was not observed between leaf discs of OX820 and WT plants, indicating that over-expression of Osa-miR820 does not impart tolerance or enhance sensitivity of the plants to salt stress.

\subsubsection{Assay for germination and seedling growth}

To follow the effect of salt stress on germination, seeds of WT plants and OX-820 lines were germinated in presence of different $\mathrm{NaCl}$ concentrations and the percentage values were plotted (Fig. 3C). Seed germination was negatively impacted in presence of salt stress and efficiency of germination decreased by 35-40\% in presence of $250 \mathrm{mM} \mathrm{NaCl}$ (Fig. 3D). At $300 \mathrm{mM} \mathrm{NaCl}$, both OX820 and WT seeds failed to germinate. This indicated that over-expression of Osa-miR820 did not influence their sensitivity towards salt.
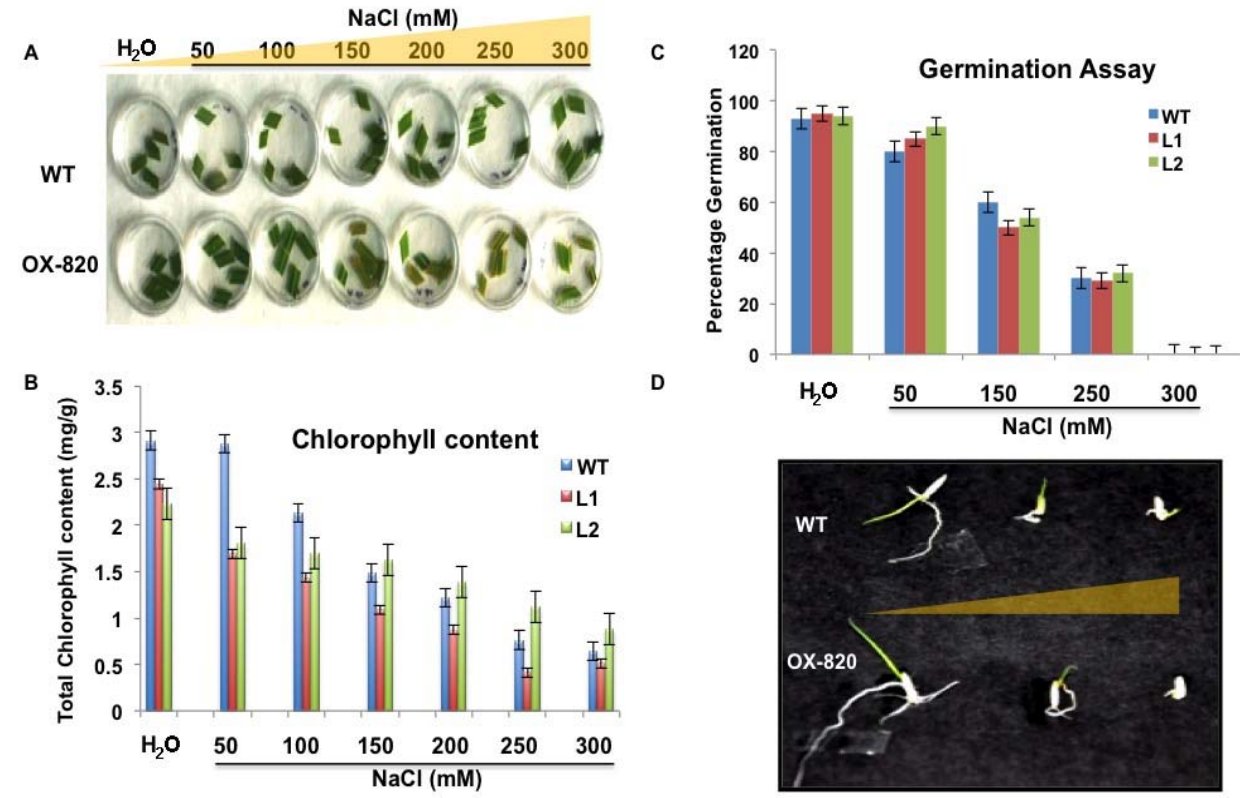

Figure 3. Assaying the effect of salt stress on OX-820 and WT plants (A) Representative picture of leaf discs placed in increasing concentration of $\mathrm{NaCl}$ solution to show chlorophyll retention. (B) Plot of chlorophyll content estimated from leaf discs after $72 \mathrm{~h}$ of stress (C) Percentage germination under increasing salt gradient (D) Representative picture showing phenotype of germinating OX-820 and WT seeds in presence of water, $150 \mathrm{mM} \mathrm{NaCl}$ and $250 \mathrm{mM} \mathrm{NaCl}$.

The growth performance of OX-820 seedlings under salt stress was also analysed, by measuring the total fresh weight (Fig. 4A), shoot length (Fig. 4B) and root length (Fig. 4C) of seedlings stressed with $\mathrm{NaCl}$ for 1 week. Under unstressed conditions (water grown) both the WT and transgenic seedlings grew well. The OX-820 seedlings were longer than WT and accumulated higher fresh weight. In the presence of $\mathrm{NaCl}$ the inhibition in growth of OX-820 seedlings was similar to the levels observed in WT. Seedling growth was severely retarded at $\mathrm{NaCl}$ concentrations higher than $200 \mathrm{mM}$. Thus, OX-820 seedlings like their WT counterparts could tolerate mild salinity conditions, but could not resist high salt concentrations.

\subsubsection{Salt stressed $O X-820$ plants resemble un-stressed WT plants}

To understand the response of plants to long term salt stress, the OX-820 and WT plants were grown till maturity by supplying $250 \mathrm{mM} \mathrm{NaCl}$ solution once every 15 days. The plants grown in 
normal watered pots served as controls. The plants were assessed for various morphological, physiological and grain yield parameters.

Under control conditions, OX-820 plants were taller with more number of tillers as compared to WT. Under salt stressed conditions, the OX-820 plants showed marked reduction in plant height, but there was no significant change in the number of tillers (Fig. 5A). In contrast the salt stressed WT plants did not show reduction in plant height but the number of primary tillers were drastically reduced. On analysing the phenotype of mature roots it was observed that under control conditions, OX-820 plants had profusely branched roots and enhanced root biomass as compared to WT plants (Fig. 5B). Exposure to salt stress reduced the root biomass in both WT and OX-820 plants.

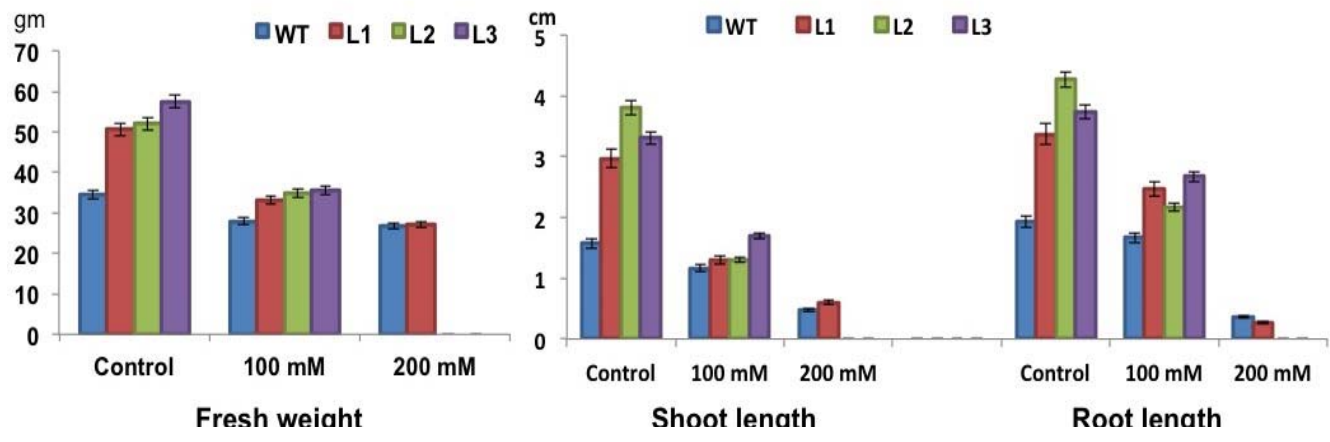

Figure 4. Effect of salt stress on OX-820 and WT seedlings. The graphs show measurements of (A) fresh weight $(\mathrm{B})$ shoot length and $(\mathrm{C})$ root length. The unit of measurement of weight is grams and unit of measurement of length is centimetre.

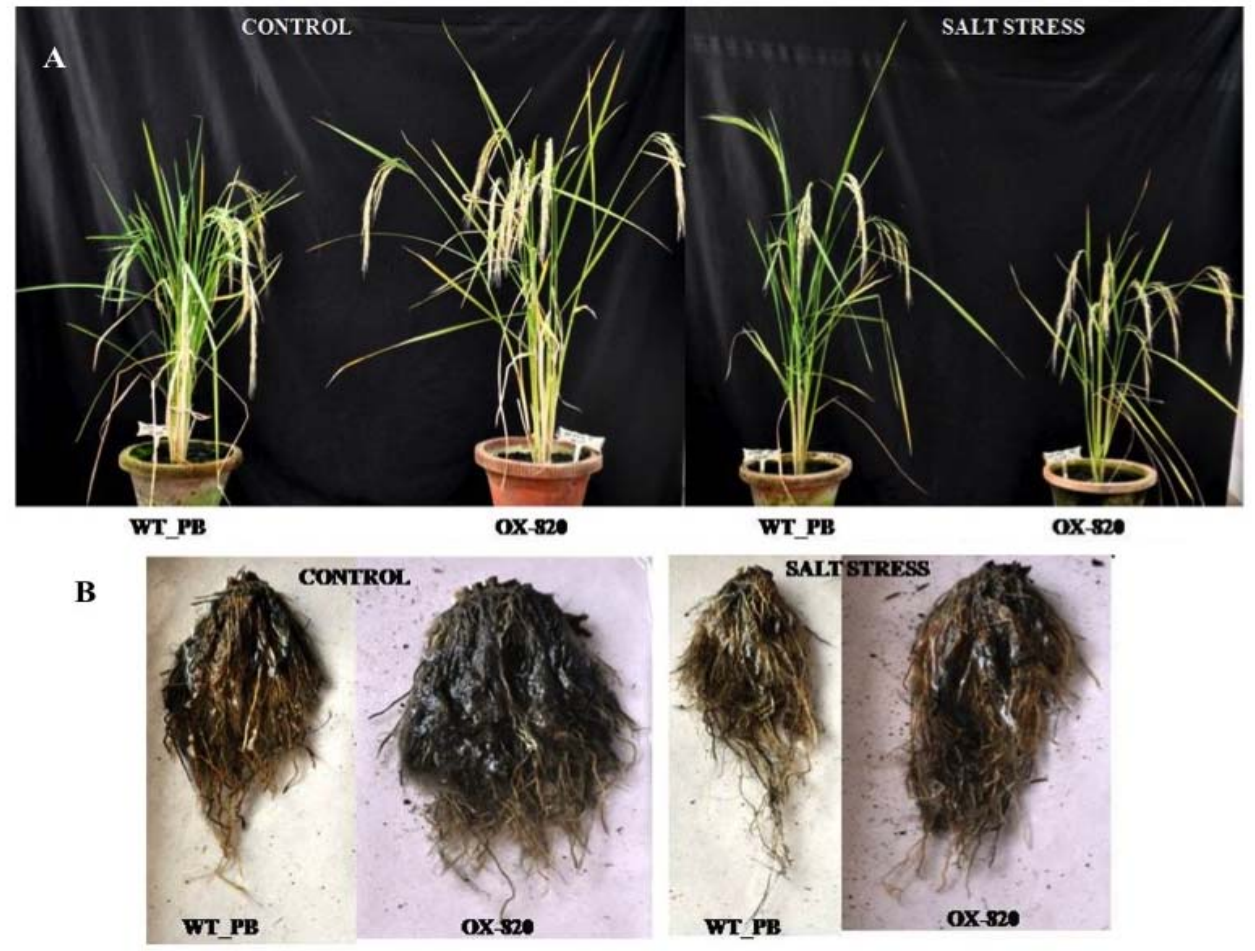

Figure 5. Phenotype of OX-820 and WT plants under long term salt stress (A) mature plants (B) roots $\mathrm{NaCl}$. 
The OX-820 plants were negatively affected by salt stress, but the extent of reduction was much less than that observed in salt stressed WT plants. Interestingly the overall salt stressed phenotype of OX-820 plants appeared similar to that of unstressed WT plants (Fig. 5). This is also apparent from the measurements for plant height (Fig. 6A), leaf length (Fig. 6B), biomass dry weight (Fig. 6C), primary tiller numbers (Fig. 6D), total number of panicles per plant (Fig. 6E) and total panicle weight per plant (Fig. 6F).
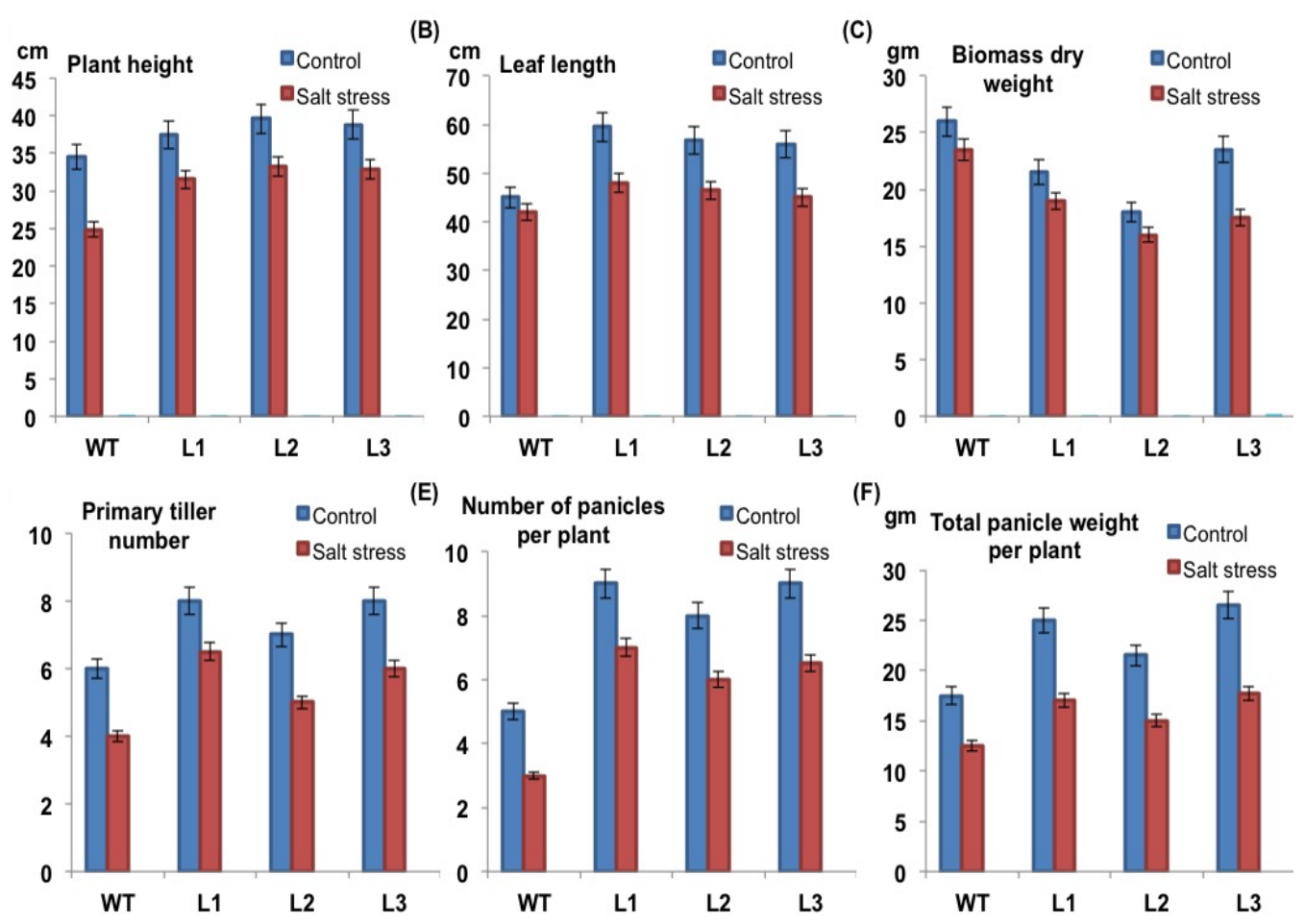

Figure 6. Comparative analysis of vegetative growth and grain yield parameters of mature OX-820 and WT plants grown under normal and salt stress conditions (A) plant height (B) leaf length (C) biomass dry weight (D) primary tiller number (E) number of panicles per plant and (F) total panicle weight per plant.

\section{3. $\mathrm{OX}-820$ plants are better adapted to long term salt stress}

To understand the effect of salt stress on physiology of OX-820 plants their photosynthetic efficiency and gas exchange measurements were recorded and analysed. Infra Red Gas Analysis (IRGA) of $\mathrm{CO}_{2}$ is most widely used, reliable, accurate and simple method for measuring gas exchange and related characteristics. Plant performance was analysed by calculating photochemical efficiency (Fv/Fm) of PSII (Fig. 7A), quantum yield (Fig. 7B), stomatal conductance (Fig. 7C) and transpiration rate (Fig. 7D). Quantum yield is the actual efficiency of light utilization during photosynthesis, representing the number of moles of $\mathrm{CO}_{2}$ fixed per mole quantum of light absorbed by a leaf [45] .

The Fv/Fm ratios of OX-820 and WT leaves were similar under control and salt stress conditions (difference of 0-0.5 is considered negligible). The imposition of stress caused a slight decrease in Fv/Fm ratios in both cases. The quantum yield, stomatal conductance and transpiration rates for $\mathrm{OX}$ 820 plants were higher as compared to the WT under unstressed conditions. In salt stressed OX-820 plants these values declined and became comparable to those of unstressed WT plants (Fig. 7B-D). The transgenic lines showed better photosynthetic and water use efficiency over the WT, which indicates that OX-820 were better adapted to retain water during salt stress. This could be one of the reasons behind their improved performance under long term salt stress. 
Free proline measurement is a well studied parameter for measuring the salt stress tolerance capacity in plants [46]. The OX-820 plants exhibited higher proline levels as compared to WT under unstressed conditions (Fig. 8). Exposure to salt stress caused a sharp decline in proline content, but the levels were relatively higher in OX-820 plants. Higher free proline content in salt stressed OX-820 transgenics indicates the activation of a protective cellular machinery that helps the plants to withstand the toxic effect of salt.

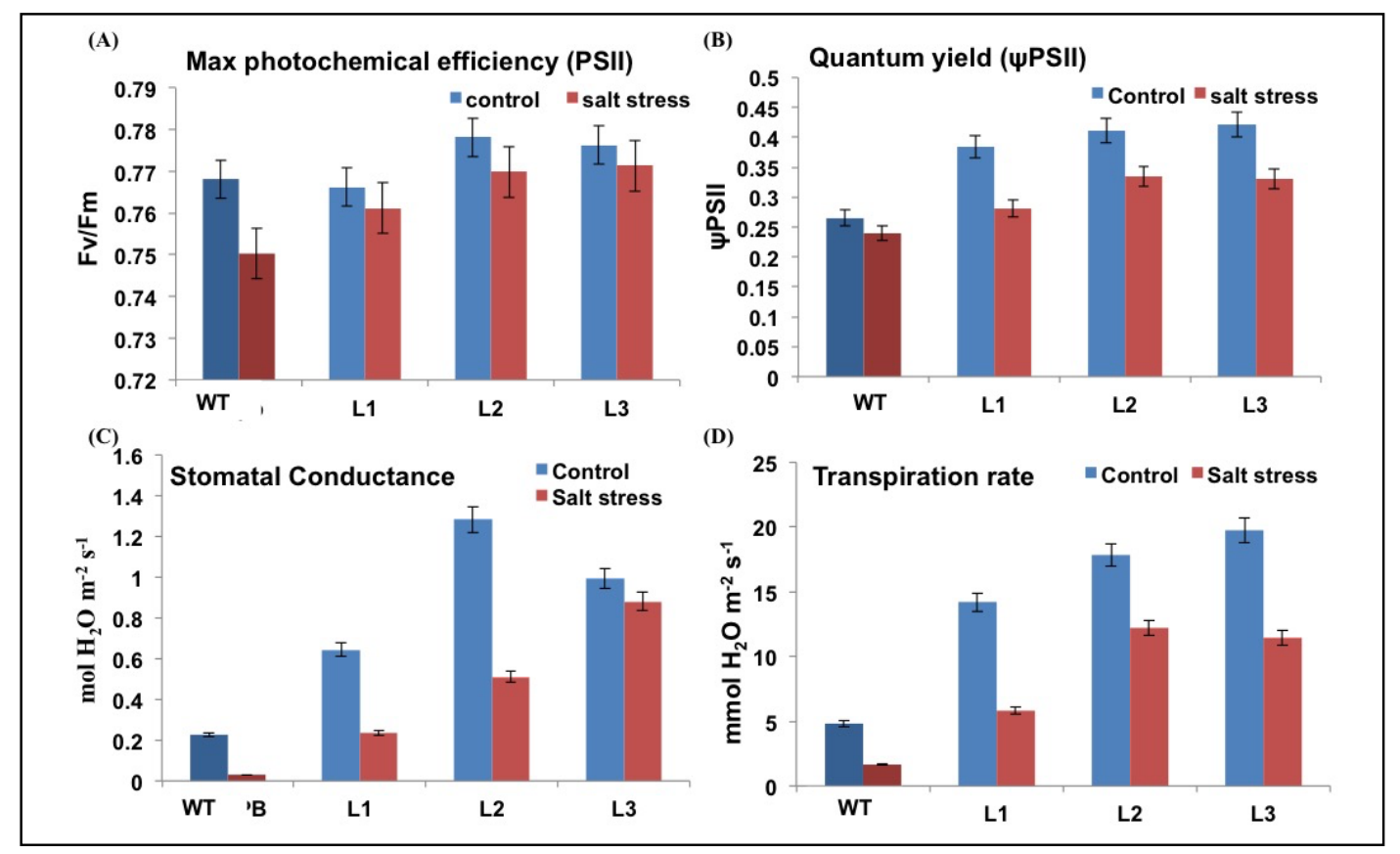

Figure 7. Comparative analysis of photosynthetic and gaseous exchange parameters of mature OX-820 and WT plants (A) Fv/Fm (B) quantum yield (C) Stomatal conductance (D) transpiration rate.

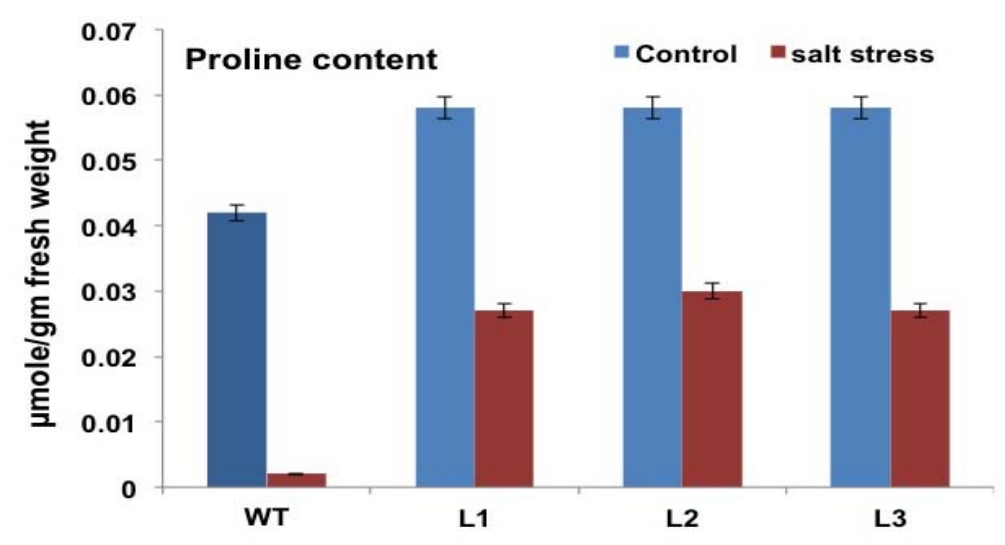

Figure 8. Measurement of free proline content in OX-820 and WT plants

\section{Discussion}

Cataloguing of miRs in rice is an important step towards understanding the physiology of this important cereal crop. A large number of miRs have been discovered in rice through experimental 
and computational approaches but functional significance of majority of them is still unknown. OsamiR820 constitutes a young, rice specific miR family and its three closely related members, OsamiR820a (MT0005263), Osa-miR820b (MT0005264) and Osa-miR820c (MI0005265) are most conserved in all Oryza AA genomes. In addition, at least 52 novel members of this family have been identified across Oryza AA genomes and 11 from Oryza punctate [38]. Size of the miR820 family varies from 1 to 16 across the Oryza AA genomes [38] and there are three members in the indica genome [36]. These three loci generate both 21-nt and 24-nt length variants by the action of the DCL-1 and DCL-3, respectively [39], suggesting the operation of complex regulatory loops by the miR family.

To gain rapid indications on the functions of this miR, the three pri-miR820(a,b,c) loci were integrated with known rice QTLs. Among the co-mapped QTLs, two QTLs associated with the traits 'Days to heading' and 'Plant height' showed interaction with all the three loci. The data obtained indicated that prospective roles of this miR family might be related with regulation of plant vigour and yield. Artificial miR based strategy was adopted to constitutively over-express the 21-nt length variants of Osa-miR820 [40]. This generated a distinct phenotype as OX-820 seedlings and mature plants were relatively longer and healthier as compared to WT. Each OX-820 rice plant had 7-8 primary tillers and robust panicles. Individual OX-820 panicles were longer, profusely branched on their primary and secondary axis and exhibited $25-30 \%$ more spikelets per panicle. It can be speculated that the robust morphology of OX-820 roots may be providing improved regulation of nutrient and water absorption from the soil for its better survival. The study of primary grain yield components revealed that in OX-820 plants the panicle weight was augmented due to 50-60\% increase in grain filling. Thus, OX-820 plants exhibited improved grain filling and enhancement in total grain yield. These observations indicated a crucial role for this miR in modulating plant architecture.

The enhanced vigor and higher grain yield attained by OX-820 plants could broadly explain the association of Osa-miR820 loci with QTLs for traits such as panicle length, culm length and leaf area to spikelet ratio. In general, high yielding varieties have a higher spikelet to leaf ratio indicating higher source to sink capacity for such cultivars [43,47]. It was evident that overexpression of OsamiR820 increased the level of branching in plants which served as a major determinant for the enhanced tillers, root biomass and panicle vigour. Morphogenetic processes in rice plants are sequential and highly synchronized such that tiller buds arise at the apical end after the formation of flag leaf. The profuse branching pattern of OX-820 plants indicates that Osa-miR820 may be acting upstream in the regulatory cascades.

The miRs not only regulate plant growth and development but also enable the plants to cope up with stressful environment. Soil salinity is one of the most damaging stresses that negatively impacts overall growth and performance of rice, though varietal differences may be observed [48-50]. Salt susceptible cultivars usually show reduced biomass and low crop yields whereas salt tolerant ones may thrive better with less yield penalties, during salinity stress. Earlier studies on salt susceptible Pusa Basmati 1 and salt tolerant Pokkali rice plants had showed that Osa-miR820 was downregulated under salt and drought stress [36]. Assays for leaf disc chlorophyll retention, germination and seedling growth indicated that over-expression of Osa-miR820 does not impart tolerance or enhance sensitivity of the plants to salt stress. The OX-820 plants, like their WT counterparts, could tolerate mild salinity conditions, but could not resist high salt concentrations.

Analysis of various vegetative and agronomic parameters revealed that the salt stressed OX-820 plants appeared similar to those of unstressed WT plants. Under salt stress, the transgenic lines displayed better photosynthetic performance as indicated by higher values of photochemical efficiency (PSII), and quantum yield. The plants also had improved water relations as indicated by the measurement of stomatal conductance and transpiration rate. Salt stress creates a physiological water deficit leading to stomatal closure thereby affecting the net transpiration rates per unit area of leaf [51,52], therefore stomatal conductance is inversely proportional to stress. The OX-820 plants also exhibited higher proline levels as compared to WT. Proline is a well known osmo-protectant that 
accumulates in plants during stress and aids in maintenance of membrane integrity, protein stability and cellular homeostasis during adverse environments [53,54]. These observations indicate that OsamiR820 regulated nodes can prime tolerance to salt stress in an agronomically advantageous manner.

The Osa-miR820 negatively regulates a DNA cytosine methyltransferase, OsDRM2 (domains rearranged methyltransferase 2) to prevent de novo methylation of C-5 residue of cytosine in the rice genome [39]. This plays a major role in curtailing the transposons and epigenetic activity during reproductive development and stress [55,56]. EST analysis also identified a putative NAC domain containing protein 77 (N77) and a putative aquaporin, plasma membrane intrinsic protein 1-5 (PIP15), as targets of Osa-miR820 [56]. The Arabidopsis homolog of DRM2 (uniprot id: Q9M548) has been well characterized. On searching the putative interacting partners of AtDRM2 using 'IntAct' [57], a database repository containing information on protein interactions, eight interacting proteins were identified. These included AGO4, AGO9, MSI4 (WD-40 repeat containing protein), HDT3 (histone deacetylase 3), TRP2 (topless related protein 2), SKP1A (SKP1-like protein 1A), H2B6 (Histone H2B.6) and ZOP1 (Zinc Finger Protein 1) protein. MSI4 is a core histone binding protein that positively regulates flowering time by promoting transcriptional repression of FLC, the flowering repressor gene [58]. HDT3 is a histone deacetylation protein whose over-expression leads to increased salt and drought tolerance in Arabidopsis [59]. TRP2 is a member of the protein family that modulates gene expression in multiple processes such as stress response, hormone signalling including auxin and jasmonic acid signalling, as well as flowering [60,61]. It may play a role in meristem specification in plants. Another important interacting partner the SKP1-A is a subunit of E3 ligase and a part of the SCF complex that is involved in ubiquitination and subsequent degradation of proteins. It indirectly influences diverse biological processes including auxin signalling pathway, vegetative and floral organ development, light signalling, embryogenesis and post embryonic development [62-64]. These reports suggest that Osa-miR820 might be indirectly manipulating important cellular mechanisms in rice through its target OsDRM2.

\section{Conclusion}

It can be concluded that over-expression of 21-nt Osa-miR820 in rice increased the plant length and level of branching, which served as major factors for their improved overall productivity by boosting tillering and augmenting panicle vigour. The OX-820 plants exhibited improved photosynthetic performance, water use efficiency and proline content. Though this did not substantially improve the tolerance of plants to salt stress, but in comparison to WT plants the overall plant biomass and tillers were relatively less affected. This manifested in a higher net panicle weight leading to a lowering of yield penalty. Therefore, the complex genetic loops regulated by the miR family modulate plant architecture and prime tolerance to salt stress in an agronomically advantageous manner. The molecular understanding of the underlying mechanisms needs to be further studied for the functional dissection of roles played by Osa-miR820.

\section{Materials and Methods}

\subsection{Plant Material and stress treatment}

Rice plants were grown under controlled conditions at $28 \pm 2{ }^{\circ} \mathrm{C}$ temperature, $70 \%$ relative air humidity and 16/8-h light/dark cycle. Seeds were collected from the WT (control) and OX-820 (artificial miR820 overexpressing)) T0 and T1 transgenics. For each analysis, 10-15 mature dehusked rice seeds of each line were surface sterilized and used. $\mathrm{NaCl}$ solution for specified concentrations were used to provide salt stress. For providing long term salt stress, the plants were supplied with $250 \mathrm{mM} \mathrm{NaCl}$ solution once every 15 days till maturity. The plants grown in normal watered pots served as controls.

\subsection{Measurement of growth and yield related parameters}


At maturity, rice plants were harvested and various grain yield parameters were measured. In each set 6 independent transgenic lines (T0 and T1) were used to record each parameter and standard deviation was calculated for each of them. 10-15 transgenic (PCR confirmed) seedlings from each of these lines were screened and grown. For each line, six plants were subjected to similar recordings under control and salt stressed conditions. The p-test values results shows the significant difference between WT and transgenic lines.

\subsection{QTL mapping}

A list of QTLs mapping to rice chromosomes 1, 7 and 10 were downloaded from Gramene database [65] and these were re-mapped to the rice chromosome using rice genome TIGR ver 7. The overlapping co-ordinates between the miR820 locus and the downloaded sequences led to the identification of putative QTLs associated with Osa-miR820 loci. Based on co-ordinate overlaps, the largest and smallest QTLs on each chromosome were also identified. The workflow for QTL mapping is described in Supplementary Fig S1. A visual interaction map was plotted for all the miR820 associated QTLs using Cytoscape 2.8.3 [66].

\subsection{Germination and seedling growth}

The assay was performed with seeds obtained from T1 plants. For this, 2 sheets of filter paper soaked in water or $\mathrm{NaCl}$ solution were placed on the petri dish and sterilized seeds were kept on it. The filter papers were replaced at an interval of two days to prevent salt accumulation. After 10 days, the number of seeds showing the emergence of plumule and radical were recorded. The germinated OX-820 seeds were confirmed for the presence of amiRNA construct. Percentage germination of was calculated by the following formula (Equation 2):

$$
\text { Germination }(\%)=(\text { number of germinated seeds on } 10 \text { th day }) \times 100
$$

The root and shoot length of 5-10 randomly selected seedlings were measured at $7^{\text {th }}$ day after germination. Fresh weight of the same seedlings was also measured with the help of weighing balance. The OX-820 seedlings were confirmed for the presence of amiRNA construct. All the experiments were done in duplicates and repeated thrice to ensure reproducibility.

\subsection{Leaf disc assay and Chlorophyll estimation}

For this assay 6th leaf of the main culm was used to maintain uniformity. The leaf was cut into pieces and these were placed on petri dishes containing $\mathrm{NaCl}$ solution of specified concentrations. Water was used as control. The leaves were floated on the solution with their abaxial surface downwards. Leaf pieces were collected at different time intervals and their chlorophyll content was measured. For each line, six independent biological replicates were taken for different salt stress and control conditions

Extraction of chlorophyll from the leaf tissues was done as per the protocol [44]. $1 \mathrm{~g}$ leaf tissue was homogenized in liquid nitrogen and $5 \mathrm{ml}$ of $80 \%$ acetone was added to it. It was allowed to thaw at room temperature and then centrifuged at $5000 \mathrm{rpm}$ for $5 \mathrm{~min}$, at room temperature. The green coloured supernatant was collected in a fresh tube. Absorbance was recorded at both $663 \mathrm{~nm}$ (A663) and $645 \mathrm{~nm}$ (A645), using 80\% acetone as a blank. The chlorophyll content was expressed in mg per gram fresh weight using the following formula (Equation 1):

Total chlorophyll $=\mathrm{A} 663 \times 8.02+\mathrm{A} 645 \times 20.2$

\subsection{Measurement of gas exchange and photosynthetic parameters}

Infra-red gas analyzer (IRGA), a hand held portable, open flow gas exchange instrument was used to measure various gas exchange parameters between the leaf sample and the measuring air chamber. The Net photosynthetic rate (PN) and stomatal conductance (gs), and other associated 
photosynthetic parameters were recorded in fully expanded leaves using an infrared gas analyzer (IRGA, LiCor, Lincoln, NE) on a sunny day between 10:00 and 11:00. The atmospheric conditions during the measurement were photo synthetically active radiation (PAR), 1,000 $\pm 5 \mu \mathrm{mol} \mathrm{m}-2 \mathrm{~s}-1$, relative humidity $66 \pm 5 \%$, atmospheric temperature $25 \pm 2^{\circ} \mathrm{C}$ and atmospheric CO2, $355 \mu \mathrm{mol}$ mol-1. All the gas exchange parameters were calculated by the LI-6400's software ver. 6.0. For each line, six independent biological replicates were taken for both salt stress and control conditions.

\subsection{Proline estimation}

Proline was determined using the method described earlier [67]. Briefly, fresh leaf tissues were homogenized in $10 \mathrm{ml}$ sulphosalicyclic acid in ice cold bath, using a pestle and mortar. Homogenate was centrifuged at 10,000 g for $15 \mathrm{~min}$. Then $2 \mathrm{ml}$ filtrate was mixed with $2 \mathrm{ml}$ acid ninhydrin and 2 $\mathrm{ml}$ glacial acetic acid. The mixture was incubated at $100^{\circ} \mathrm{C}$ for $1 \mathrm{~h}$ until the coloured complex is developed in water bath. The reaction was terminated by rapid cooling on ice. Then $4 \mathrm{ml}$ toluene was added to the coloured complex and reaction mixture was mixed by vortexing for 15-20 s. Optical density of layer with chromophore (red-pink in colour) was read at $520 \mathrm{~nm}$. Proline content was estimated by using L-Proline standard curve.

\section{Supplementary Materials}

Supplementary Figure 1 The workflow for QTL mapping.

\section{Authors' contributions}

Conceptualization, N.S.-M.; Methodology, N.S.; Software, N.S.; Validation, N.S. and S.K.; Formal Analysis, N.S. and S.K.; Investigation, N.S. and S.K.; Resources, N.S.-M.; Writing - Original Draft Preparation, N.S.; Writing - Review \& Editing, N.S.-M.; Supervision, N.S.-M.; Project Administration, N.S.-M.; Funding Acquisition, N.S.-M.

\section{Funding}

NSM is thankful for the funds received from International Centre for Genetic Engineering and Biotechnology (ICGEB), New Delhi and Department of Biotechnology (DBT), Government of India.

All authors have approved the final manuscript for publication.

\section{Data Availability Statement}

The data presented in this study are available on request from the corresponding author.

\section{Acknowledgements}

Authors thank Mr. Sandeep Panchal for help with raising the transgenics. NS and SK are thankful to Council of Scientific and Industrial Research (CSIR), India, for providing research fellowship.

\section{Conflict of Interest:}

The authors declare no conflict of interest.

\section{Competing Financial Interests:}

The authors declare no competing financial interests. 


\section{References}

1. Colaiacovo, M.; Lamontanara, A.; Bernardo, L.; Alberici, R.; Crosatti, C.; Giusti, L.; Cattivelli, L.; Faccioli, P. On the complexity of miRNA-mediated regulation in plants: novel insights into the genomic organization of plant miRNAs. Biol. Direct 2012, 7(1), 15.

2. Mittal, D.; Mukherjee, S. K.; Vasudevan, M.; Sanan-Mishra, N. Identification of tissue-preferential expression patterns of rice miRNAs. J. Cell. Biochem. 2013, 114, 2071-2081.

3. Li, C.; Zhang, B. MicroRNAs in control of plant development. J. Cellular Physiol. 2016, 231(2), 303313.

4. Yang, T.; Wang, Y.; Teotia, S.; Zhang, Z.; Tang, G. The making of leaves: how small RNA networks modulate leaf development. Front Plant Sci. 2018, 9, 824

5. Song, X.; Li, Y.; Cao, X.; Qi, Y. MicroRNAs and their regulatory roles in plant-environment interactions. Annu. Rev. Plant Biol. 2019, 70, 489-525.

6. Sanan-Mishra, N.; Kumari, A. Role of RNA interference in seed germination. In Plant small RNA: biogenesis, regulation and applications. Guleria, P.; Kumar, V.; Eds.; Elsevier, 2020; pp. 101-110.;

7. Chiou, T. J.; Aung, K.; Lin, S.I.; Wu, C.C.; Chiang, S.F.; Su, C.L. Regulation of phosphate homeostasis by microRNA in Arabidopsis. Plant Cell 2006, 18, 412-421.

8. Mittal, D.; Sharma, N.; Sharma, V.; Sopory, S.K.; Sanan-Mishra, N. Role of microRNAs in rice plant under salt stress. Ann. Appl. Biol. 2016, 168, 2-18.

9. Manavella, P.A.; Yang, S.W.; Palatnik, J. Keep calm and carry on: miRNA biogenesis under stress. Plant J. 2019, 99(5), 832-843.

10.Huang, S.Q.; Peng, J.; Qiu, C.X.; Yang, Z.M. Heavy metal-regulated new microRNAs from rice. J Inorg Biochem. 2009, 103, 282-287.

11. Li, T.; Li, H.; Zhang, Y. X.; Liu, J. Y. Identification and analysis of seven $\mathrm{H}(2) \mathrm{O}(2)$-responsive miRNAs and 32 new miRNAs in the seedlings of rice (Oryza sativa L. ssp. indica). Nucleic Acids Res 39, 2821-33 (2011)

12.Liu, Q.; Zhang, H. Molecular identification and analysis of arsenite stress-responsive miRNAs in rice. J Agric Food Chem. 2012, 60, 6524-6536.

13. Goswami K.; Mittal D.; Gautam B.; Sopory S.K.; Sanan-Mishra N. Mapping the salt stress-induced changes in the root miRNome in Pokkali rice. Biomolecules. 2020, 10(4), 498.

14.Zhu, Q.-H.; Spriggs, A.; Matthew, L.; Fan, L.; Kennedy, G.; Gubler, F.; Helliwell, C. A diverse set of microRNAs and microRNA-like small RNAs in developing rice grains. Genome Res. 2008, 18(9), 1456-1465..

15.Shen, J.; Xie, K.; Xiong, L. Global expression profiling of rice microRNAs by one-tube stem-loop reverse transcription quantitative PCR revealed important roles of microRNAs in abiotic stress responses. Mol Genet Genomics 2010, 284, 477-488.

16.Zhou, L. Liu, Y.; Liu, Z.; Kong, D.; Duan, M.; Luo, L. Genome-wide identification and analysis of drought-responsive microRNAs in Oryza sativa. J Exp Bot. 2010, 61, 4157-68.

17.Barrera-Figueroa, B.E.; Gao, L.; Wu, Z.; Zhou, X.; Zhu, J.; Jin, H.; Liu, R.; Zhu, J.K. High throughput sequencing reveals novel and abiotic stress-regulated microRNAs in the inflorescences of rice. BMC Plant Biol. 2012, 12, 132.

18. Goswami, K.; Tripathi, A.; Gautam, B.; Sanan-Mishra, N. Impact of Next Generation Sequencing in elucidating the role of microRNA related to multiple abiotic stresses. In Plant Abiotic Stress: Molecular Biology and Biotechnological Advances. Roychoudhury. A.; Tripathi, D.K. Eds.; JohnWiley and Sons Ltd.; 2019; 389-426.

19.Goswami, K.; Tripathi, A.; Sanan-Mishra, N. Comparative miRomics of salt-tolerant and saltsensitive rice. J Integrative Bioinformatics. 2017, 14(1), 20170002.

20. Kawashima, C.G.; Yoshimoto, N.; Maruyama-Nakashita, A.; Tsuchiya, Y. N.; Saito, K.; Takahashi, H.; Dalmay, T. Sulphur starvation induces the expression of microRNA-395 and one of its target genes but in different cell types. Plant J. 2009, 57(2), 313-321.

21. Yuan, N.; Yuan, S.; Li, Z.; Li, D.; Hu, Q.; Luo, H. Heterologous expression of a rice miR395 gene in Nicotiana tabacum impairs sulfate homeostasis. Sci Rep. 2016, 6, 28791. 
22. Chen, Z.; Gao, X.; Zhang, J. Alteration of osa-miR156e expression affects rice plant architecture and strigolactones (SLs) pathway. Plant Cell Rep. 2015, 34, 767-781.

23. Peng, H.; Chun, J.; Ai, T.B.; Tong, Y.A.; Zhang, R.; Zhao, M.M.; Chen, F.; Wang, S.H. MicroRNA profiles and their control of male gametophyte development in rice. Plant Mol Biol. 2012, 80(1), 85-102.

24. Zhou, L.; Liu, Y.; Liu, Z.; Kong, D.; Duan, M.; Luo, L. Genome-wide identification and analysis of drought-responsive microRNAs in Oryza sativa. J Expt Bot. 2010, 61(15), 4157-4168.

25. Xia, K.; Wang, R.; Ou, X.; Fang, Z.; Tian, C.; Duan, J.; Wang, Y.; Zhang, M. OsTIR1 and OsAFB2 downregulation via OsmiR393 overexpression leads to more tillers, early flowering and less tolerance to salt and drought in rice. PLoS One 2012, 7, e30039.

26. Jiao, Y.; Wang, Y.; Xue, D.; Wang, J.; Yan, M.; Liu, G.; Dong, G.; Zeng, D.; Lu, Z.; Zhu, X.; Qian, Q.; $\mathrm{Li}, \mathrm{J}$. Regulation of OsSPL14 by OsmiR156 defines ideal plant architecture in rice. Nature Genetics. 2010, 42(6), 541-544.

27. Pu, C.X.; Ma, Y.; Wang, J.; Zhang, Y.C.; Jiao, X.W.; Hu, Y.H.; Wang, L.L.; Zhu, Z.G.; Sun, D.; Sun, Y. Crinkly4 receptor-like kinase is required to maintain the interlocking of the palea and lemma, and fertility in rice, by promoting epidermal cell differentiation. Plant J. 2012, 70(6), 940-953.

28. Yang F.; Yu D. Overexpression of Arabidopsis MiR396 enhances drought tolerance in transgenic tobacco plants. Acta Bot Yunnanica. 2009, 31(5), 421-426.

29. Jeong, D.-H.; Park, S.; Zhai, J.; Gurazada, S.G.R.; De Paoli, E.; Meyers, B.C.; Green, P. J. Massive analysis of rice small RNAs: mechanistic implications of regulated MicroRNAs and variants for differential target RNA cleavage, Plant Cell. 2011, 23, 4185-4207.

30. Yu X.; Wang H.; Lu Y.Z.; de Ruiter M.; Cariaso M.; Prins M.; Van T.A.; He Y.K. Identification of conserved and novel microRNAs that are responsive to heat stress in Brassica rapa. J Exp Bot. 2012, 63(2), 1025-1038.

31. Zhang, S.; Xie, M.; Ren, G.; Yu, B. CDC5, a DNA binding protein, positively regulates posttranscriptional processing and/or transcription of primary microRNA transcripts. Proc Natl Acad Sci USA. 2013, 110, 17588-17593.

32. Luo, Y.C.; Zhou, H.; Li, Y.; Chen, J.Y.; Yang, J.H.; Chen, Y.Q.; Qu, L.H. Rice embryogenic calli express a unique set of microRNAs, suggesting regulatory roles of microRNAs in plant postembryogenic development. FEBS Letts. 2006, 580(21), 5111-5116.

33. Nosaka, M.; Ishiwata, A.; Shimizu-Sato, S.; Ono, A.; Ishimoto, K.; Noda, Y.; Sato, Y. The copy number of rice CACTA DNA transposons carrying MIR820 does not correlate with MIR820 expression. Plant Signal Behav. 2013, 8(8), e25169.

34. Nosaka, M.; Ono, A.; Ishiwata, A.; Shimizu-Sato, S.; Ishimoto, K.; Noda, Y.; Sato, Y. Expression of the rice microRNA miR820 is associated with epigenetic modifications at its own locus. Genes Genet Syst. 2013, 88(2), 105-112.

35. Zhang, J.W.; Long, Y.; Xue, M.D.; Xiao, X.G.; Pei, X.W. Identification of microRNAs in response to drought in common wild rice (Oryza rufipogon Griff.) shoots and roots. PloS One 2017, 12(1), e0170330.

36. Sharma, N.; Tripathi, A.; Sanan-Mishra, N. Profiling the expression domains of a rice-specific microRNA under stress. Front Plant Sci. 2015, 6, 333.

37. Sharma, D.; Tiwari, M.; Lakhwani, D.; Tripathi, R. D.; Trivedi, P. K. Differential expression of microRNAs by arsenate and arsenite stress in natural accessions of rice, Metallomics. 2015, 7, 174-187.

38. Singh, A.K.; Furtado A.; Brozynska M.; Sanan-Mishra N.; Henry R.J. Phylogeny and molecular evolution of miR820 and miR396 microRNA families in Oryza AA genomes. Trop Plant Biol. 2018, 11(1-2),1-16.

39. Nosaka, M.; Itoh, J.I.; Nagato, Y.; Ono, A.; Ishiwata, A.; Sato, Y. Role of transposon-derived small RNAs in the interplay between genomes and parasitic DNA in rice. PLoS Genet. 2012, 8(9), e1002953. 
40.Sharma, N.; Panchal, S.; Sanan-Mishra, N. Protocol for artificial microRNA mediated overexpression of miR820 in indica rice. Am J Plant Sci. 2015, 06, 1951-1961.

41. Fang, R.; Li, L.; Li, J. Spatial and temporal expression modes of MicroRNAs in an elite rice hybrid and its parental lines. Planta 2013, 238, 259-269.

42.Li, X.; Qian, Q.; Fu, Z.; Wang, Y.; Xiong, G.; Zeng, D.; Wang, X.; Liu, X.; Teng, S.; Hiroshi, F.; Yuan, M.; Luo, D.; Han, B.; Li, J. Control of tillering in rice. Nature. 2003, 422(6932), 618-621.

43. Reynolds, M.; Rajaram, S.; Sayre, K. Physiological and genetic changes of irrigated wheat in the post-green revolution period and approaches for meeting projected global demand. Crop Sci. 1999, 39, 1611-1621.

44. Allen, R. S.; Li, J.; Stahle, M. I.; Dubroué, A.; Gubler, F.; Millar, A. A. Genetic analysis reveals functional redundancy and the major target genes of the Arabidopsis miR159 family. Proc Natl Acad Sci USA.2007, 104(41), 16371-16376.

45. Long, S.P.; Zhu, X.; Naidu, S.L.; Ort, D.R. Can improvement in photosynthesis increase crop yields? Plant Cell Environ. 2006, 29, 315-330.

46. Sawahel, W.A.; Hassan, A.H. Generation of transgenic wheat plants producing high levels of the osmoprotectant proline. Biotechnol. Lett. 2002, 24, 721-725.

47.Abdoli, M.; Saeidi, M.; Jalali-Honarmand, S.; Mansourifar, S.; Ghobadi, M.-E.; Cheghamirza, K. Effect of source and sink limitation on yield and some agronomic characteristics in modern bread wheat cultivars under post anthesis water deficiency. Acta Agric Slov. 2013, 101, 173182.

48.Lutts, S.; Kinet, J.M.; Bouharmont, J. Changes in plant response to $\mathrm{NaCl}$ during development of rice (Oryza sativa L.) varieties differing in salinity resistance. J Exp Bot 1995, 46, 1843-1852.

49.Zeng, L.; Shannon, M.C. Salinity effects on seedling growth and yield components of rice. Crop Sci 2000, 40(4), 996-1003.

50.Moradi, F.; Ismail, A.; Egdane, J.; Gregorio, G. Salinity tolerance of rice during reproductive development and association with tolerance at the seedling stage. Indian J. Plant Physiol. 2003, 8, 105-116.

51.Munns, R.; Tester, M. Mechanisms of salinity tolerance. Annu Rev Plant Biol 2008, 59, 651-81.

52.Silva, E.N. da; Ribeiro, R.V.; Ferreira-Silva, S.L.; Viégas, R.A.; Silveira, J.A.G. Salt stress induced damages on the photosynthesis of physic nut young plants. Sci Agric. 2011, 68, 62-68.

53.Khedr, A.H.A. Proline induces the expression of salt-stress-responsive proteins and may improve the adaptation of Pancratium maritimum L. to salt-stress. J Exp Bot. 2003, 54, 2553-2562.

54.Xu, J.; Yin, H.; Li, X. Protective effects of proline against cadmium toxicity in micropropagated hyperaccumulator, Solanum nigrum L. Plant Cell Rep. 2009, 28, 325-333.

55. Cao, X.; Jacobsen, S.E. Role of the Arabidopsis DRM methyltransferases in de novo DNA methylation and gene silencing. Curr Biol. 2002, 12, 1138-1144.

56. Sahu, P.P.; Pandey, G.; Sharma, N.; Puranik, S.; Muthamilarasan, M.; Prasad, M. Epigenetic mechanisms of plant stress responses and adaptation. Plant Cell Rep, 2013, 32(8), 1151-1159

57.Hermjakob, H. IntAct: an open source molecular interaction database. Nuc Acids Res. 2004, 32, 452D-455.

58.He, Y. Regulation of flowering time by histone acetylation in arabidopsis. Science 2003, 302, 17511754.

59.Sridha, S.; Wu, K. Identification of AtHD2C as a novel regulator of abscisic acid responses in Arabidopsis. Plant J. 2006, 46, 124-133.

60.Causier, B.; Ashworth, M.; Guo, W.; Davies, B. The TOPLESS interactome: a framework for gene repression in arabidopsis. Plant Physiol. 2012, 158, 423-438.

61.Osmont, K.S.; Hardtke, C.S. The topless plant developmental phenotype explained! Genome Biol. 2008, 9, 219. 
62.Gray, W.M.; del Pozo, J.C.; Walker, L.; Hobbie, L.; Risseeuw, E.; Banks, T.; Crosby, W.L.; Yang, M.; Ma, H.; Estelle, M. Identification of an SCF ubiquitin-ligase complex required for auxin response in Arabidopsis thaliana. Genes Dev. 1999, 13, 1678-1691.

63.Zhao, D.; Ni, W.; Feng, B.; Han, T.; Petrasek, M. G.; \& Ma, H. Members of the Arabidopsis-SKP1like gene family exhibit a variety of expression patterns and may play diverse roles in Arabidopsis. Plant Physiol. 2003, 133, 203-217.

64.Liu, F. The ASK1 and ASK2 genes are essential for arabidopsis early development. Plant Cell Online 2004, 16, 5-20.

65. Tello-Ruiz, M.K.; Naithani, S.; Stein, J.C.; Gupta, P.; Campbell, M.; Olson, A.; Wei, S.; Preece, J.; Geniza, M.J.; Jiao, Y.; Lee, Y.K.; Wang, B.; Mulvaney, J.; Chougule, K.; Elser, J.; Al-Bader, N.; Kumari, S.; Thomason, J.; Kumar, V.; Bolser, D.M.; ... Ware, D. Gramene 2018: unifying comparative genomics and pathway resources for plant research. Nucleic Acids Res. 2018,.46(D1), D1181-D1189.

66. Shannon, P.; Markiel, A.; Ozier, O.; Baliga, N.S.; Wang, J.T.; Ramage, D.; Amin, N.; Schwikowski, B.; Ideker, T. Cytoscape: a software environment for integrated models of biomolecular interaction networks. Genome Res 2003, 13, 2498-2504.

67. Bates, L.S.; Waldren, R.P.; Teare, I.D. Rapid determination of free proline for water-stress studies. Plant Soil 1973, 39, 205-207. 


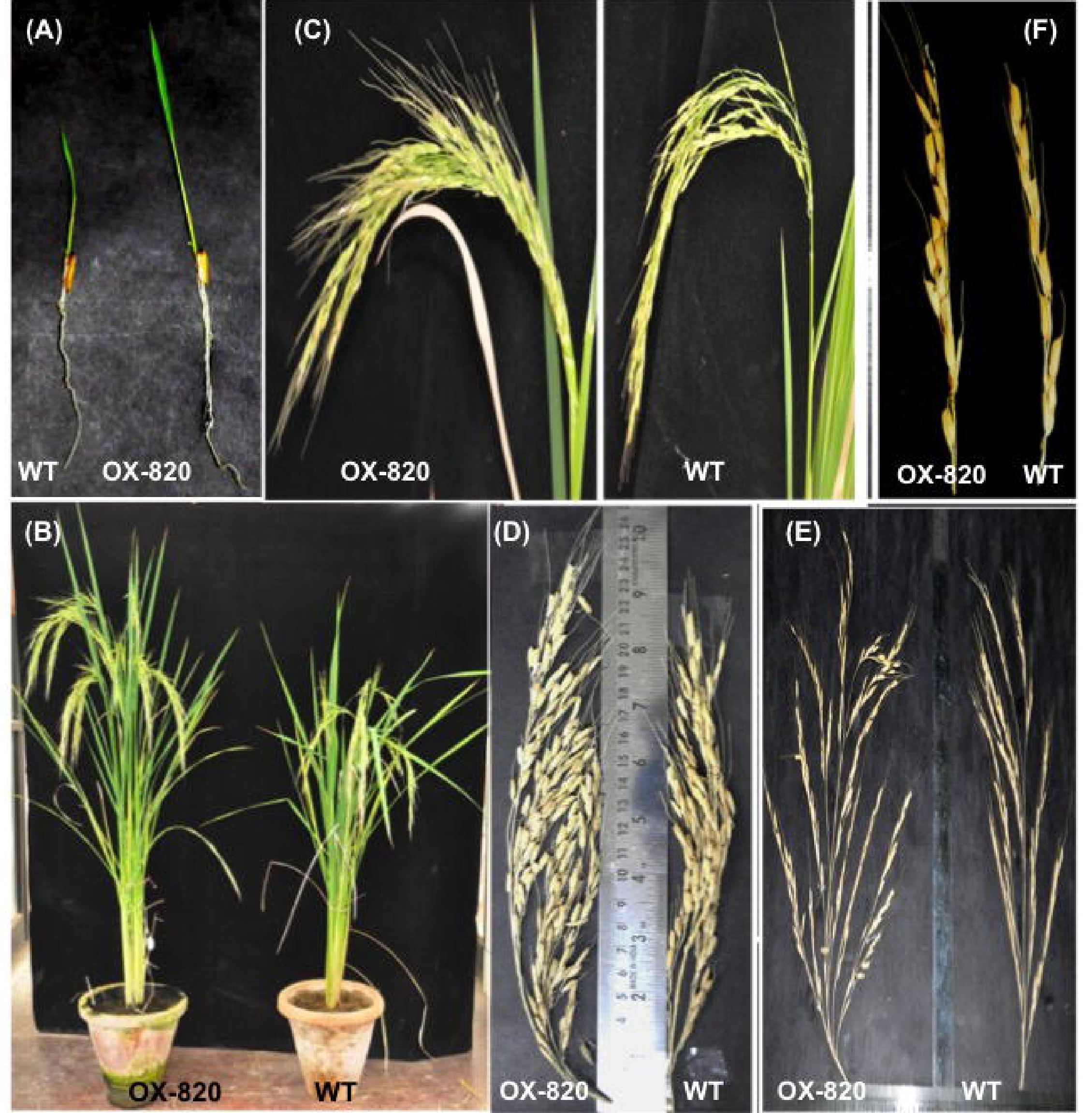




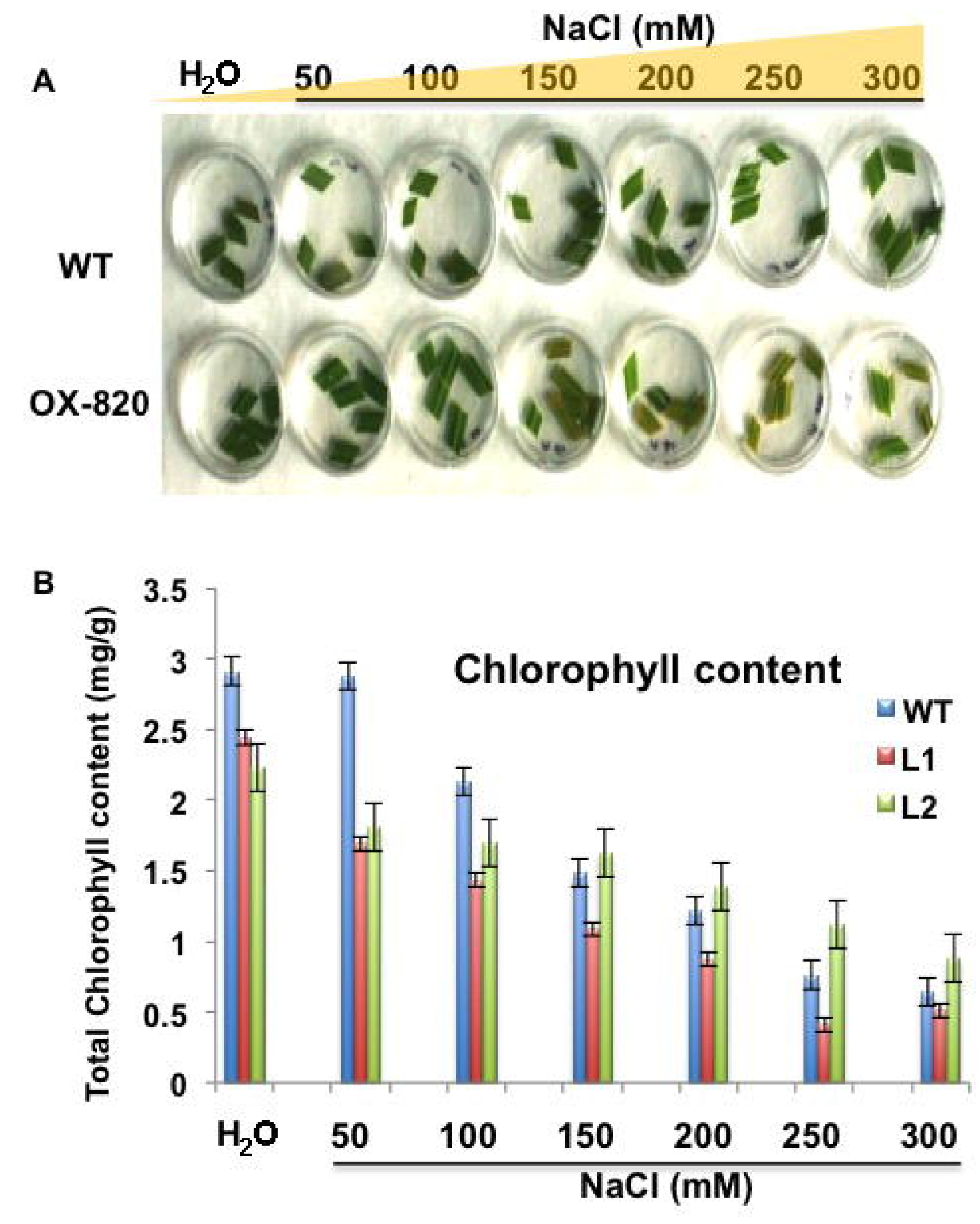

C $\quad 120$ Germination Assay

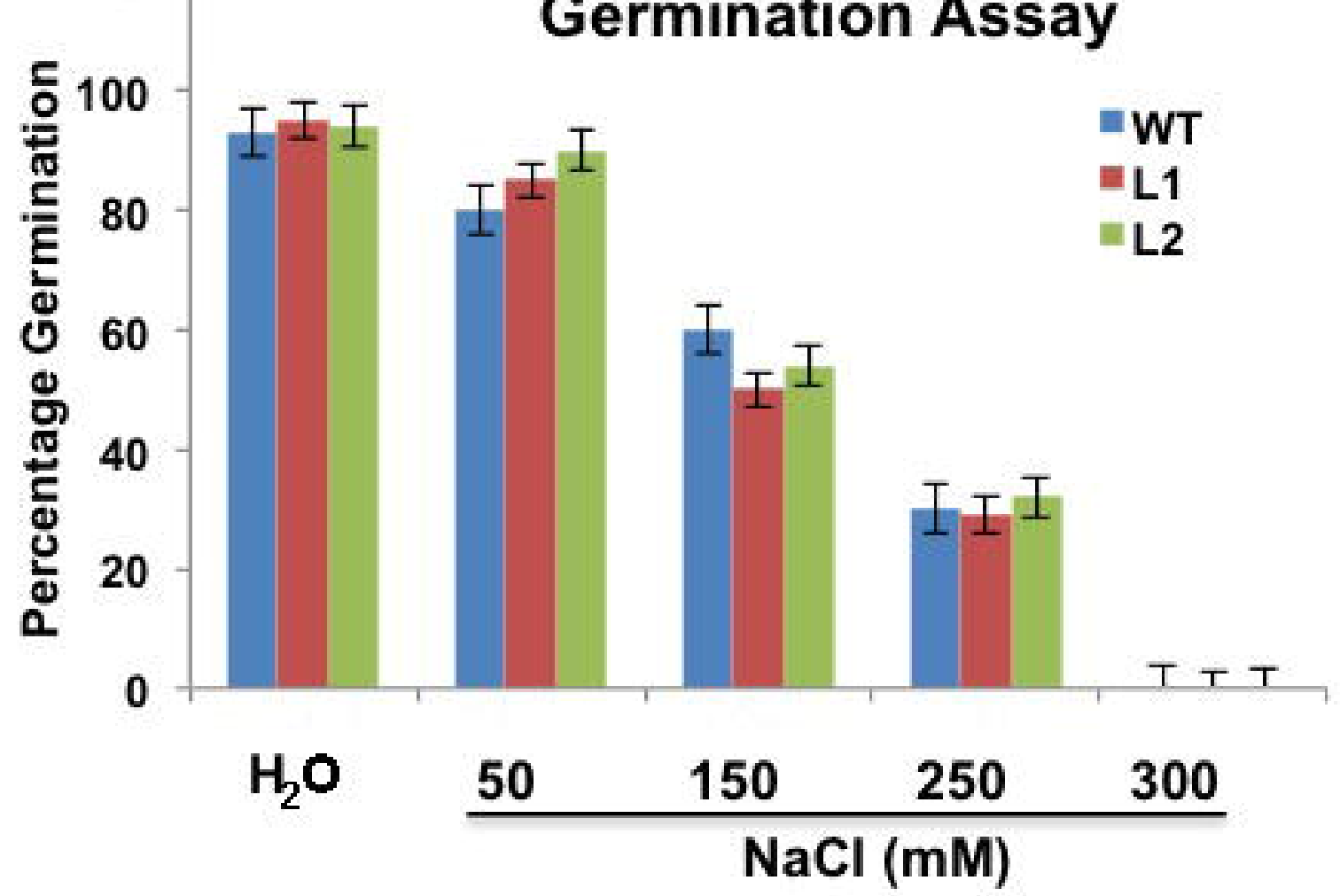

D

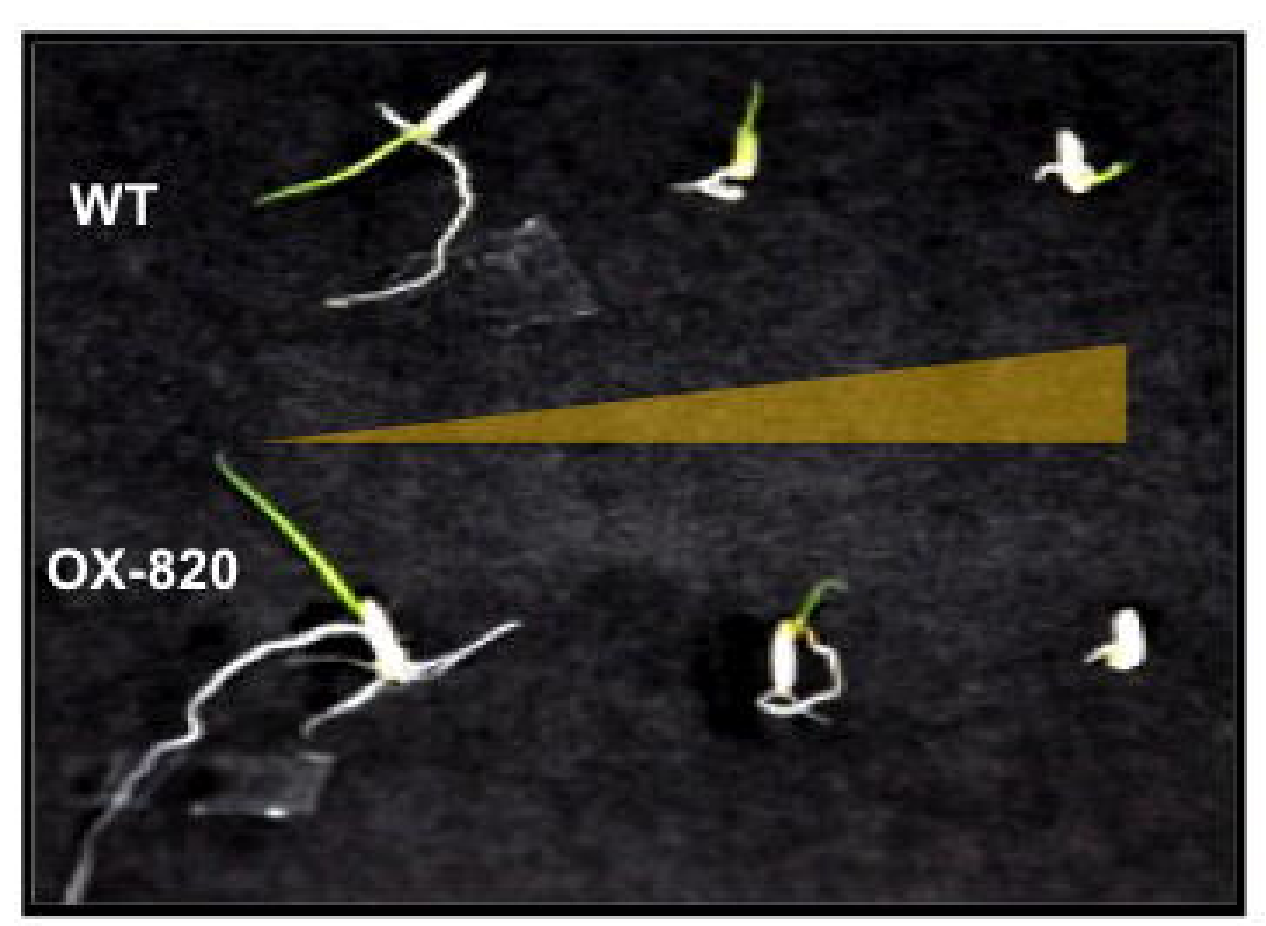




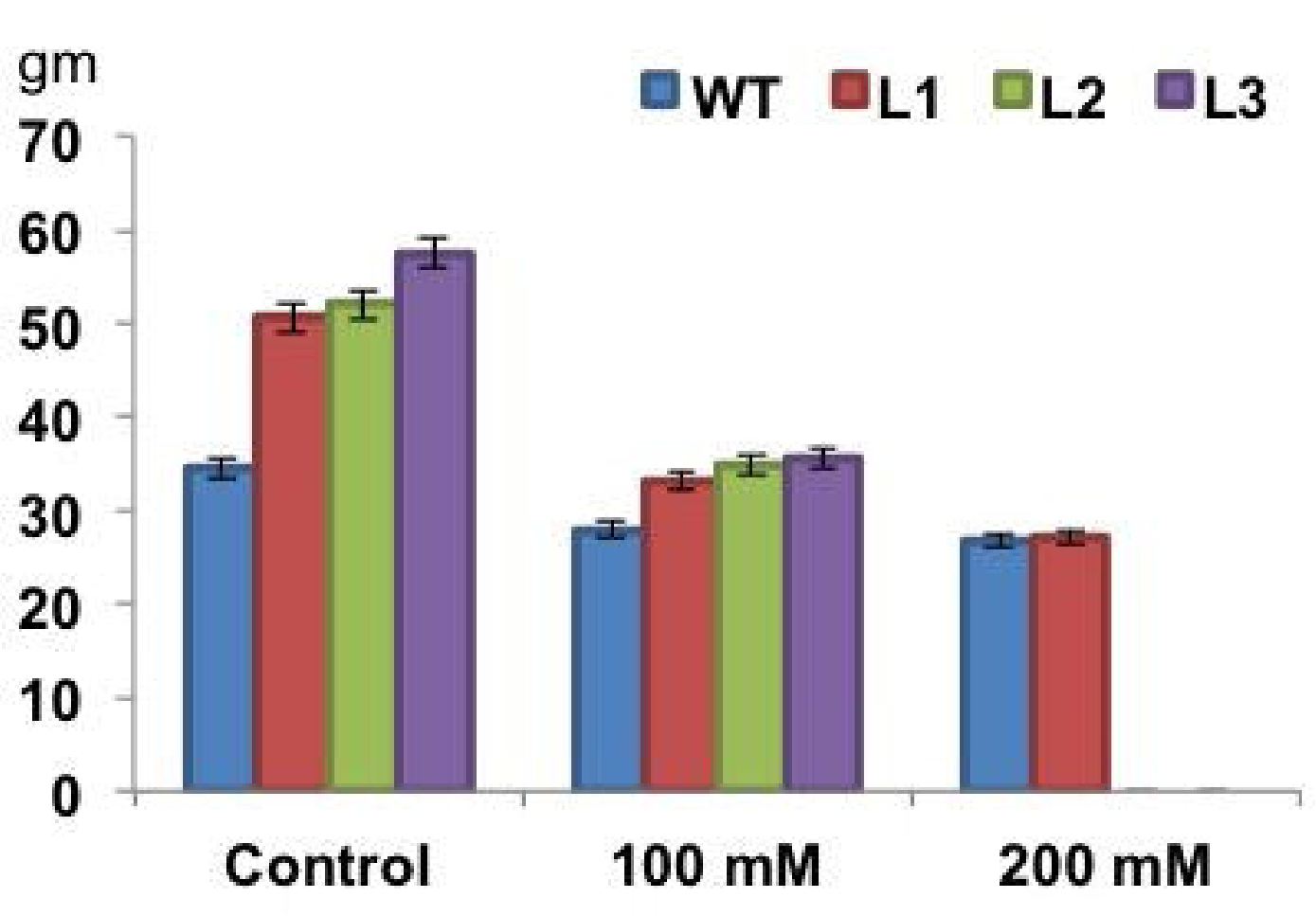

Fresh weight

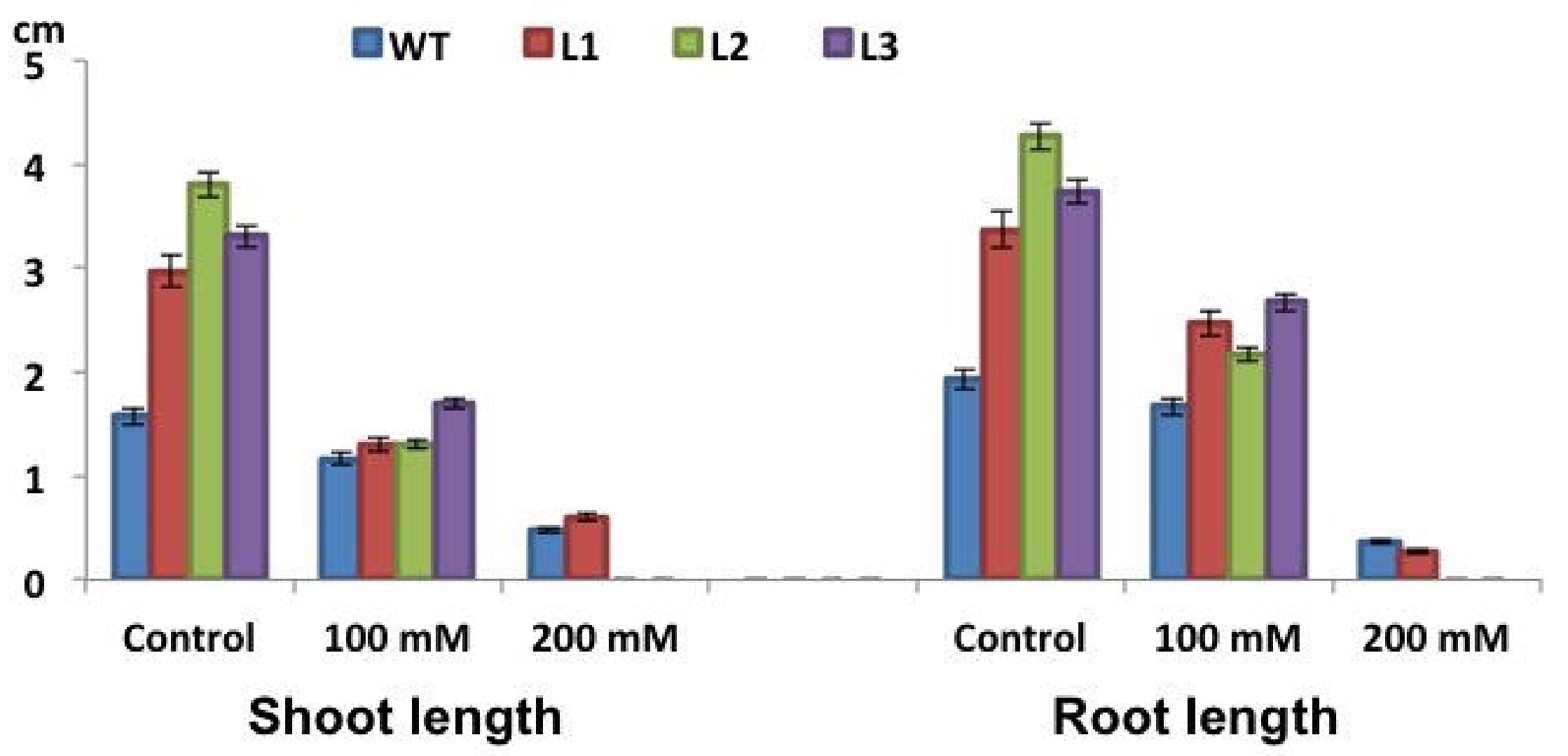




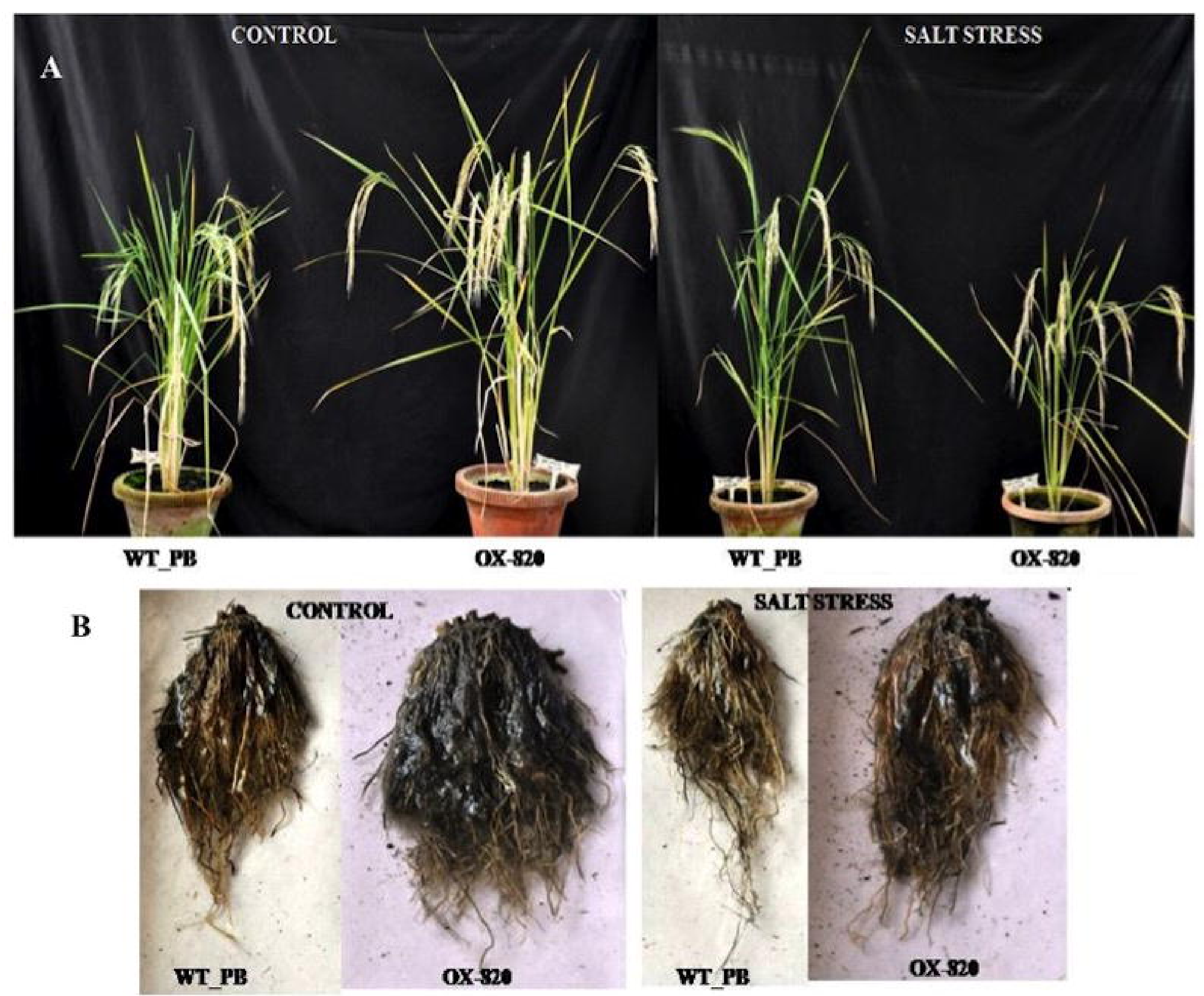




$$
(\mathrm{A})
$$

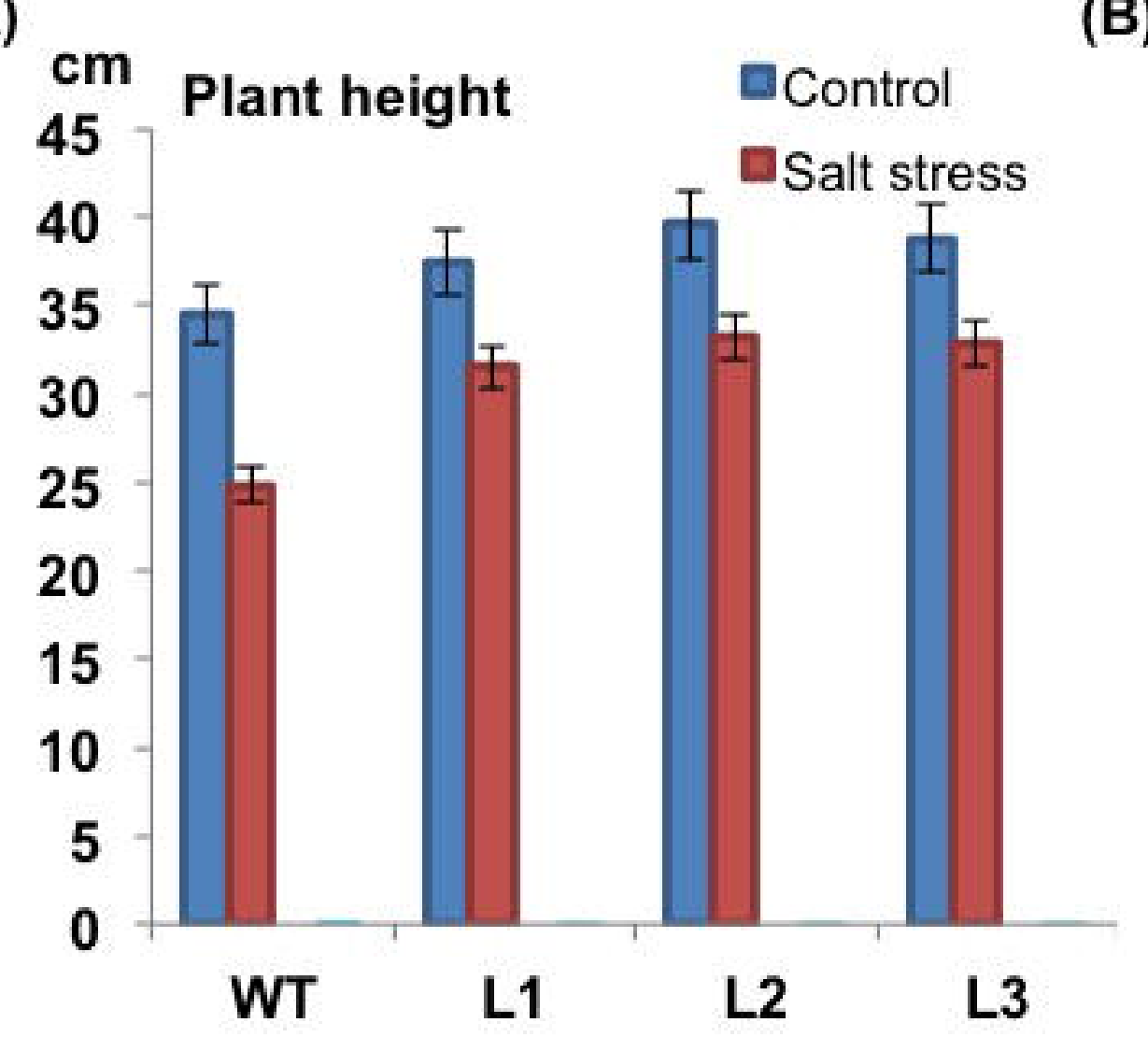

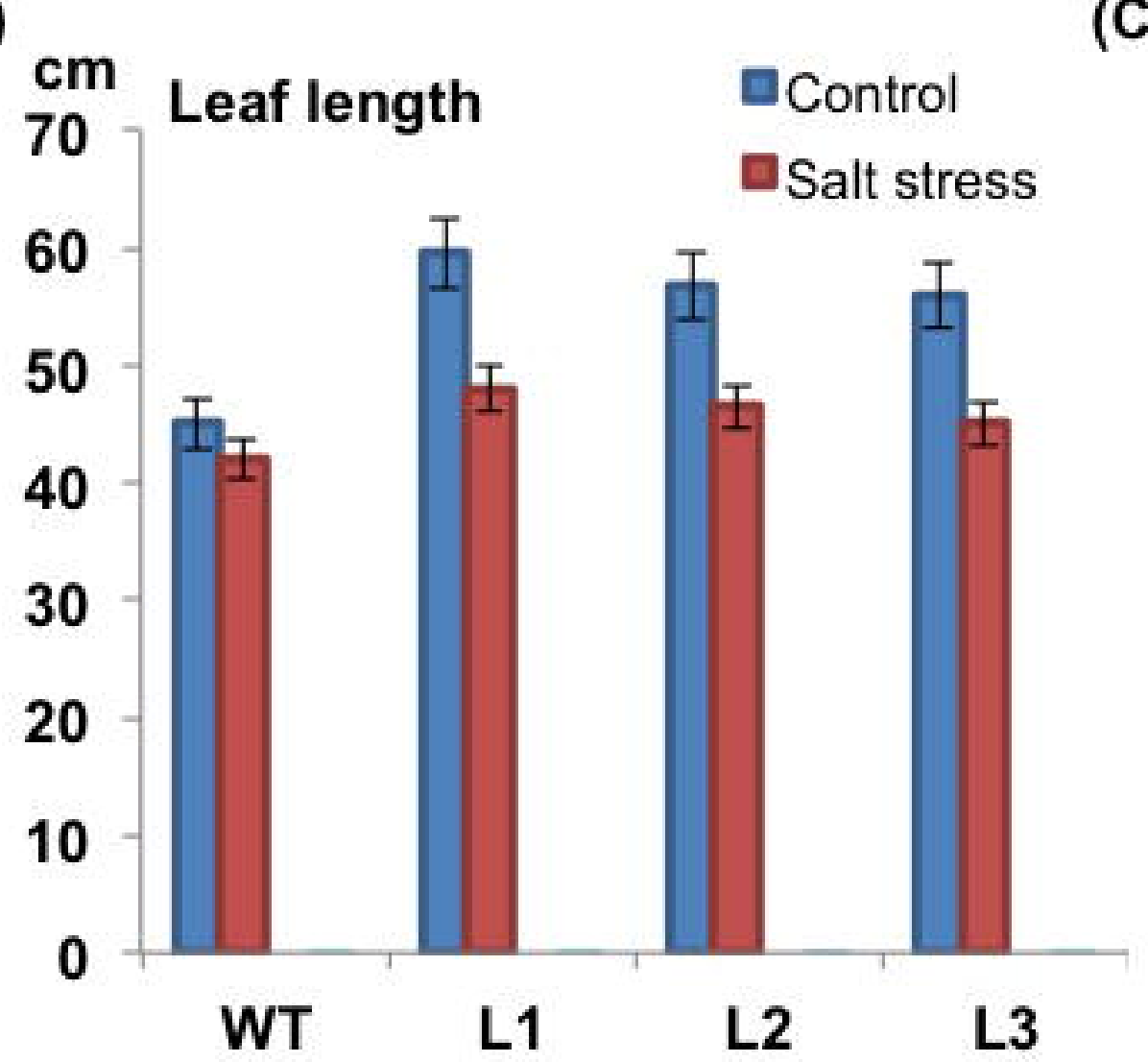

(E) Number of panicles

-Control
(C)

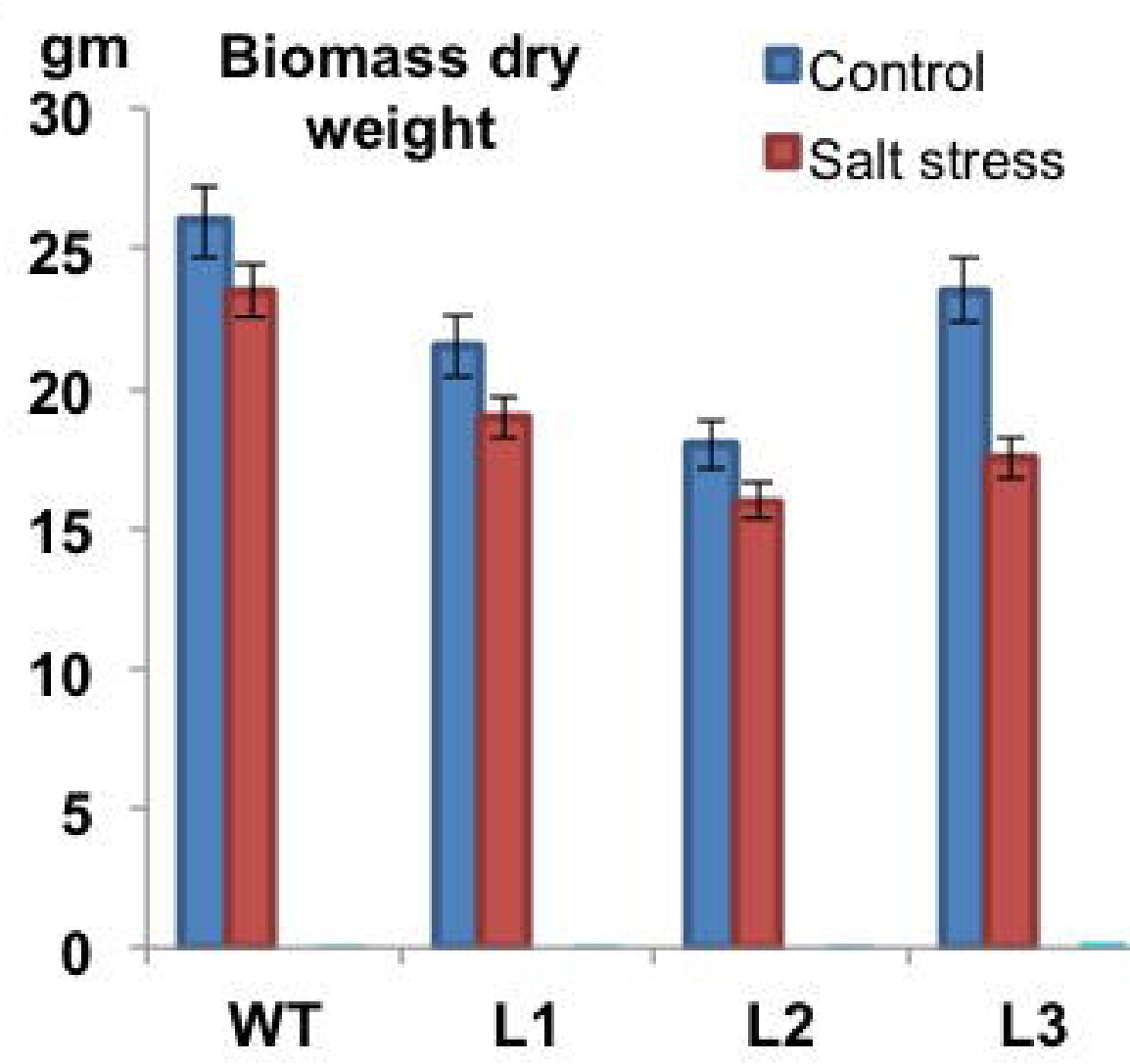

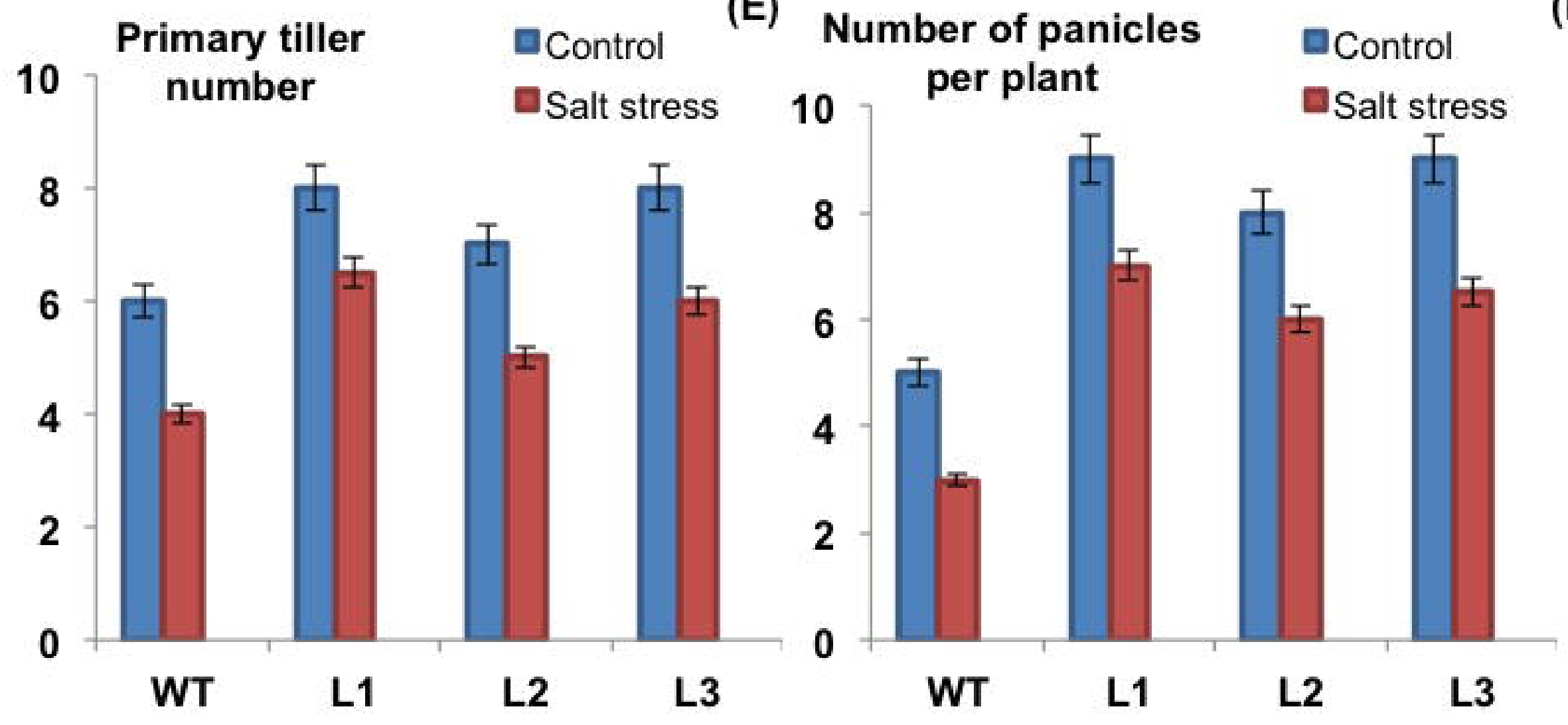

(D)
(F)

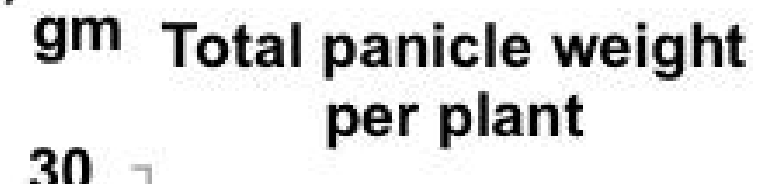

-Control

- Salt stress

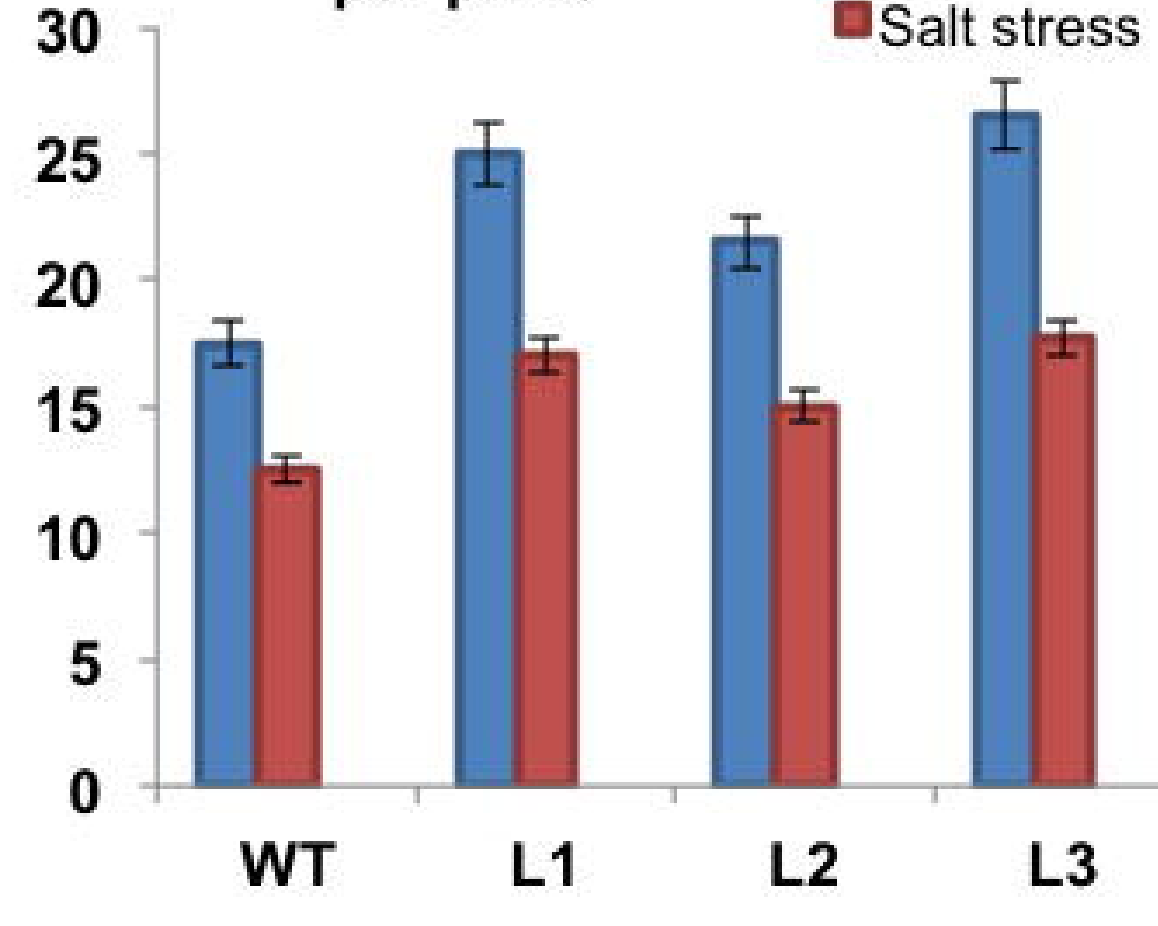




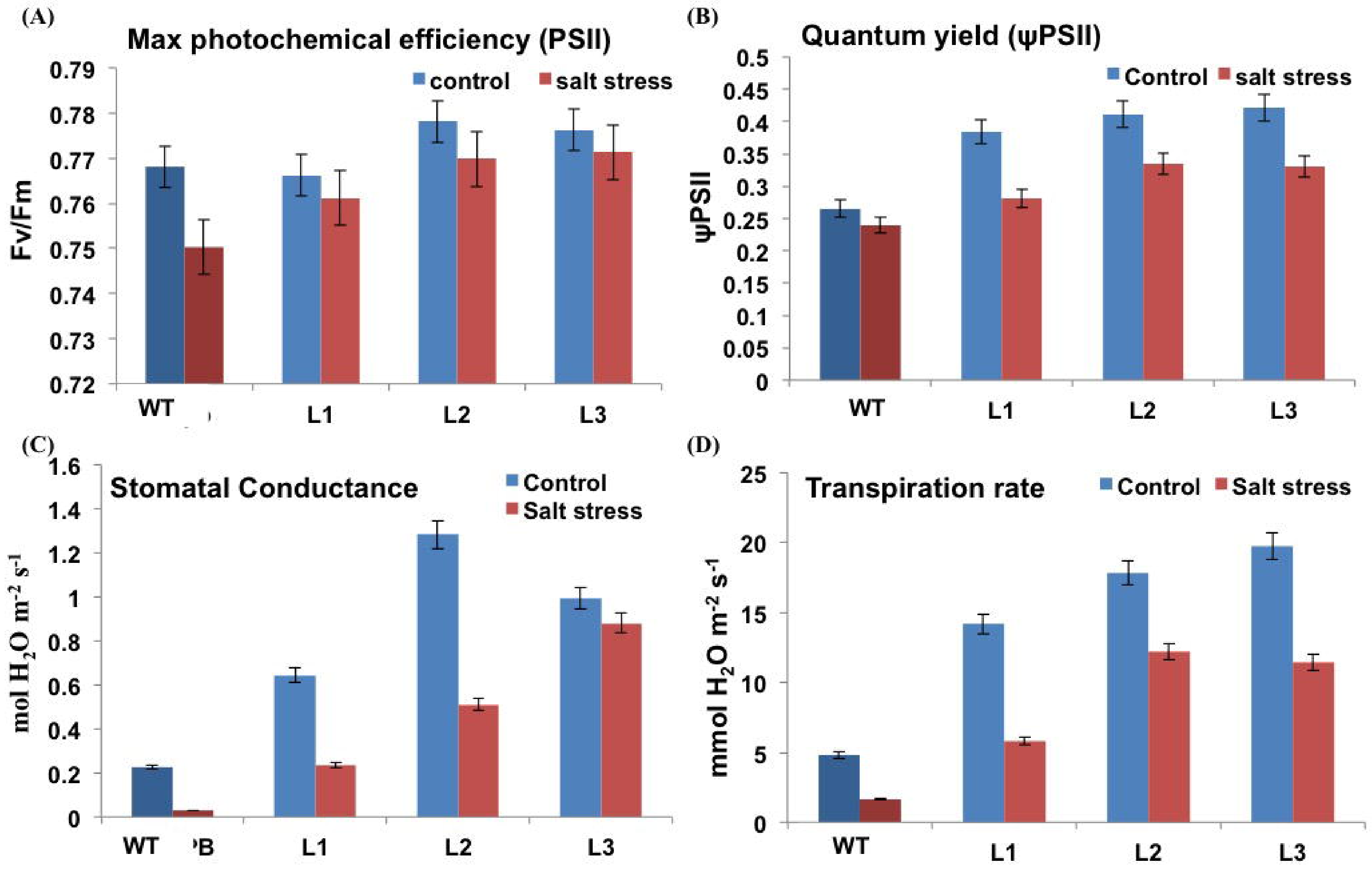




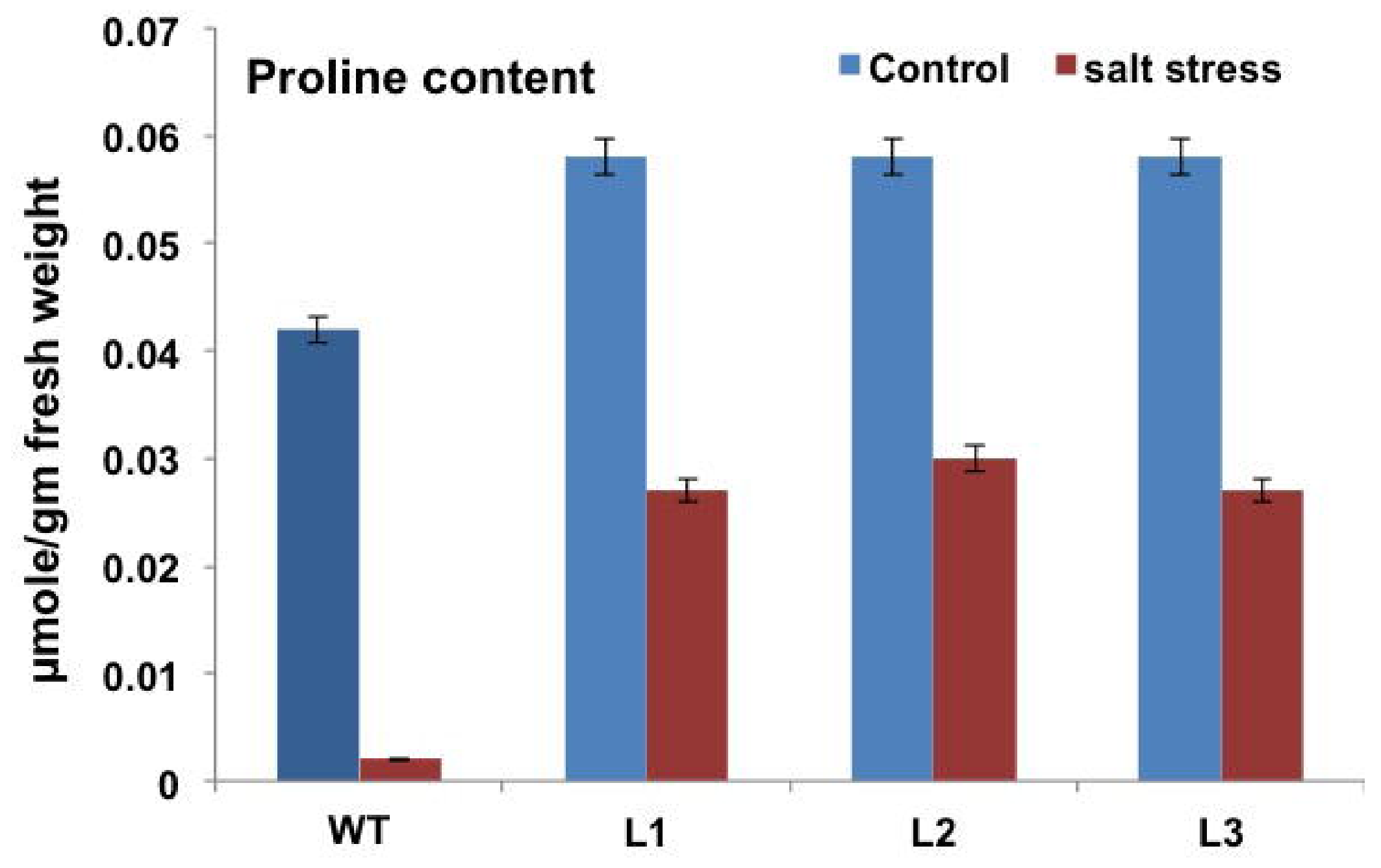

